\title{
GENERALIZED HUNTER-SAXTON EQUATION AND THE GEOMETRY OF THE GROUP OF CIRCLE DIFFEOMORPHISMS
}

\author{
BORIS KHESIN, JONATAN LENELLS, AND GERARD MISIOŁEK
}

\begin{abstract}
We study an equation lying 'mid-way' between the periodic HunterSaxton and Camassa-Holm equations, and which describes evolution of rotators in liquid crystals with external magnetic field and self-interaction. We prove that it is an Euler equation on the diffeomorphism group of the circle corresponding to a natural right-invariant Sobolev metric. We show that the equation is bihamiltonian and admits both cusped as well as smooth traveling-wave solutions which are natural candidates for solitons. We also prove that it is locally well-posed and establish results on the lifespan of its solutions. Throughout the paper we argue that despite similarities to the $\mathrm{KdV}, \mathrm{CH}$ and $\mathrm{HS}$ equations, the new equation manifests several distinctive features that set it apart from the other three.
\end{abstract}

\section{Contents}

1. Introduction

2. Eulerian nature of the $\mu \mathrm{HS}$ equation

2.1. Geodesic flow on the group of circle diffeomorphisms

2.2. $\mu \mathrm{HS}$ as an evolution equation

3. Variational principles and physical motivation

3.1. $\mu \mathrm{HS}$ as a bi-variational equation

3.2. Physical motivation

4. Hamiltonian framework and conservation laws

4.1. Bihamiltonian structure and the $\mu \mathrm{HS}$ hierarchy

4.2. Relation with bi-variational principles

5. The periodic Cauchy problem

5.1. Local well-posedness and persistence result

5.2. Global solutions with non-negative angular momentum density

5.3. Breakdown of classical solutions

6. Traveling-wave solutions

6.1. Solitary waves

6.2. Traveling waves on the circle

7. Geometry of the group of circle diffeomorphisms

7.1. Christoffel symbols and the covariant derivative

7.2. Sectional curvature

8. $\mu \mathrm{HS}$ as a bi-hamiltonian system on the Virasoro group 


\section{INTRODUCTION}

The equation we study in this paper is

$$
-u_{t x x}=-2 \mu(u) u_{x}+2 u_{x} u_{x x}+u u_{x x x}
$$

where $u=u(t, x)$ is a time-dependent function on the unit circle $S^{1}=\mathbb{R} / \mathbb{Z}$ and $\mu(u)=\int_{S^{1}} u d x$ denotes its mean. The closest relatives of this equation are the Camassa-Holm equation

$$
u_{t}-u_{t x x}+3 u u_{x}=2 u_{x} u_{x x}+u u_{x x x}
$$

and the Hunter-Saxton equation

$$
-u_{t x x}=2 u_{x} u_{x x}+u u_{x x x}
$$

both of which have recently attracted a lot of attention among the integrable systems and the PDE communities. The Camassa-Holm equation, originally derived in $[\mathrm{FF}]$ as an abstract equation with a bihamiltonian structure, was introduced independently in [CH] as a shallow water approximation]. The Hunter-Saxton equation first appeared in [HS] as an asymptotic equation for rotators in liquid crystals. We show that $\mu \mathrm{HS}$ can be viewed as a natural generalization of the rotator equation once we allow interactions of rotators and an external magnetic field.

In order to compare equation $\mu \mathrm{HS}$ ) with the other two equations we first recall a few facts about the latter. It is known that for spatially periodic functions $(\overline{C H})$ is the geodesic equation on the infinite-dimensional group $\mathcal{D}^{s}\left(S^{1}\right)$ of orientation-preserving diffeomorphisms of the unit circle $S^{1} \simeq \mathbb{R} / \mathbb{Z}$ of (sufficiently high) Sobolev class $H^{s}$ and endowed with a right-invariant metric given at the identity by the $H^{1}$ inner product, see [M],

$$
\langle u, v\rangle_{H_{1}}=\int_{S^{1}}\left(u v+u_{x} v_{x}\right) d x .
$$

Equation ( $(\mathrm{CH})$ is a completely integrable system with a bihamiltonian structure and hence it possesses an infinite family of commuting Hamiltonian flows, as well as an associated sequence of conservation laws, see e.g. [CM] for the periodic case. Furthermore, it admits soliton-like solutions (called peakons) in both the periodic and the non-periodic setting, see [CH].

Equation $(\overline{\mathrm{HS}})$, on the other hand, describes the geodesic flow on the homogeneous space of the group $\mathcal{D}^{s}\left(S^{1}\right)$ modulo the subgroup of rigid rotations $\operatorname{Rot}\left(S^{1}\right) \simeq S^{1}$ equipped with the $\dot{H}^{1}$ right-invariant metric, which at the identity is

$$
\langle u, v\rangle_{\dot{H}_{1}}=\int_{S^{1}} u_{x} v_{x} d x .
$$

This equation possesses a bihamiltonian structure as well, see e.g. [HZ, KM].

In Section 2 we will show that the $\mu \mathrm{HS}$ equation has a similar geometric origin: it describes the geodesic flow on $\mathcal{D}^{s}\left(S^{1}\right)$ with the right-invariant metric given at the identity by the inner product

$$
\langle u, v\rangle=\mu(u) \mu(v)+\int_{S^{1}} u_{x} v_{x} d x .
$$

(see also Section 5). One motivation for considering this inner product comes from the observation made in [L2] that there is a natural isometry between $\mathcal{D}^{s}\left(S^{1}\right) / S^{1}$ equipped with the $\dot{H}^{1}$ metric and an open subset $U$ of the (infinite-dimensional) unit sphere in $L^{2}\left(S^{1}\right)$. Consequently, the group $\mathcal{D}^{s}\left(S^{1}\right)$ can be viewed as an $S^{1}$ bundle over $U$ with $\langle\cdot, \cdot\rangle$ inducing the simplest possible metric in this bundle which

\footnotetext{
${ }^{1}$ For an interesting discussion of the attendant physical principles see $\mathrm{J}$.
} 
projects to the $\dot{H}^{1}$ metric on the base $U$. (In a sense, this picture can be thought of as an infinite-dimensional version of the Hopf $S^{1}$-fibration.) Thus equation $\mu \mathrm{HS}$ as a geodesic equation for $\mathcal{D}^{s}\left(S^{1}\right)$ with this metric is expected to have the 'nicest' properties among the integrable equations related to this group, owing to constant sectional curvature of the base $U$ and the 'constant' metric along the fibers $S^{1}$.

In Section 3 we will present two variational principles for the $\mu \mathrm{HS}$ equation and show how it arises as an asymptotic rotator equation in a liquid crystal with a preferred direction if we take into account the reciprocal action of dipoles on themselves. The bihamiltonian structure of $(\mu \mathrm{HS})$ together with its attendant infinite hierarchy of conservation laws will be described in Sections 4 and 8.

In spite of the presence of the nonlocal expression $\mu(u)$ the $\mu \mathrm{HS}$ equation is a welldefined PDE (rather than a mixed integro-differential equation)2. This is because the mean of any solution $u$ is constant in time and hence is completely determined by the initial condition. In light of this fact the term $2 \mu(u) u_{x}$ may appear rather harmless and might be expected to have only a minor influence on the phenomenology of the equation. However, in Section [5] we shall see that it has a strong effect on well-posedness of the associated Cauchy problem in that it is responsible for $\mu \mathrm{HS}$ admitting global (in time) solutions. In the same section a blow-up mechanism for $\mu \mathrm{HS}$ solutions will also be described. In this respect the $\mu \mathrm{HS}$ equation resembles (CH) rather than $(\overline{\mathrm{HS}})$ as all classical solutions of the periodic HS equation except space-independent solutions blow up in finite time, see [G] or [Y]. Let us point out that just like the breaking waves of (프 $)$ can be continued as global weak solutions (see [BC2]) the solutions of $(\underline{\mathrm{HS}})$ can also be extended to all times (see [BC] or [L3] for the periodic case).

Furthermore, whereas ( $\underline{\mathrm{HS}}$ ) does not have any bounded traveling-wave solutions at all, the $\mu \mathrm{HS}$ equation will be shown in Section 6 to admit traveling waves (in the periodic case) that can be regarded as the appropriate candidates for solitons 3

In Section 7 we compute the sectional curvature of $\mathcal{D}^{s}\left(S^{1}\right)$ and in Section 8 we revisit the derivation of the $\mu \mathrm{HS}$ equation on the Virasoro group and describe its two compatible Poisson structures.

Finally, we remark that although our focus in this paper is on the periodic case many of the arguments presented here can be adapted, with obvious modifications, to study the corresponding equation on the real line.

\section{EULERIAN NATURE OF THE $\mu$ HS EQUATION}

2.1. Geodesic flow on the group of circle diffeomorphisms. Let $\mathcal{D}^{s}\left(S^{1}\right)$ denote the set of circle diffeomorphisms of a sufficiently high Sobolev class $H^{s}$ and let $T \mathcal{D}^{s}\left(S^{1}\right)$ be its tangent bundle 4 Consider the following inner product on the corresponding Lie algebra of vector fields, that is, on the tangent space $T_{i d} \mathcal{D}^{s}\left(S^{1}\right)=$

\footnotetext{
${ }^{2}$ We mention here that a related (nonperiodic) equation obtained by replacing $\mu(u)$ by a constant has appeared previously as a special case of a model derived in [MN] for the propagation of surface waves in an ideal fluid with surface tension, and whose soliton solutions were studied using tools of algebraic and complex geometry in $\mathrm{ACHM}$.

${ }^{3}$ That (HS) does not admit any bounded traveling wave solutions is related to the fact that the equation itself is defined on the homogeneous space $\mathcal{D}^{s}\left(S^{1}\right) / S^{1}$, i.e. 'modulo rotations' and hence one considers all solutions modulo traveling.

${ }^{4}$ In the bulk of the paper we can assume that the Sobolev index $s>3 / 2$ is simply large enough for the computations to make sense (for example, taking $s=\infty$, which corresponds to the Fréchet topology on smooth functions, will do). In Section 5 on well-posedness, where analytical details are of essence, we will be more precise.
} 
$\left\{u(x) \partial / \partial x: u \in H^{s}\left(S^{1}\right)\right\}:$

$$
\langle u, v\rangle=\mu(u) \mu(v)+\int u_{x} v_{x} d x=\int u A v d x,
$$

where the inertia operator $A=\mu-\partial_{x}^{2}$ acts on a function $v$ by $A v=\mu(v)-v_{x x}$. Using right translations we extend this inner product to a right-invariant metric on the group $\mathcal{D}^{s}\left(S^{1}\right)$ so that

$$
\langle U, V\rangle_{\eta}=\int U \eta_{x} d x \int V \eta_{x} d x+\int \frac{U_{x} V_{x}}{\eta_{x}} d x
$$

for any diffeomorphism $\eta \in \mathcal{D}^{s}\left(S^{1}\right)$ and any $U, V \in T_{\eta} \mathcal{D}^{s}\left(S^{1}\right)$.

Theorem 2.1. The $\mu H S$ equation is an Euler equation on $\mathcal{D}^{s}\left(S^{1}\right)$. More precisely, it corresponds to the equation of the geodesic flow on $\mathcal{D}^{s}\left(S^{1}\right)$ with respect to the rightinvariant metric (2.2).

Remark 2.2. Observe that $A$ is invertible as an operator from $H^{s}$ onto $H^{s-2}$ with the inverse $A^{-1} w=u$ given explicitly by

$$
\begin{aligned}
u(x)=\left(\frac{x^{2}}{2}-\frac{x}{2}+\frac{13}{12}\right) \mu(w) & +\left(x-\frac{1}{2}\right) \iint_{0}^{y} w(s) d s d y \\
& -\int_{0}^{x} \int_{0}^{y} w(s) d s d y+\iint_{0}^{y} \int_{0}^{s} w(r) d r d s d y .
\end{aligned}
$$

Moreover, note that $A^{-1}$ commutes with $\partial_{x}$ and $\mu(u)=\mu(A u)$.

Proof of Theorem 2.1. Using (2.1) and the definition of the coadjoint operator

$$
\left\langle a d_{v}^{*}(u), w\right\rangle=\langle u,[v, w]\rangle,
$$

where $[v, w]=v_{x} w-v w_{x}$, we readily compute

$$
a d_{v}^{*}(u)=A^{-1}\left(2 v_{x} A u+v A u_{x}\right) .
$$

It follows that the corresponding Euler equation is

$$
u_{t}=-a d_{u}^{*}(u)=-A^{-1}\left(2 \mu(u) u_{x}-2 u_{x} u_{x x}-u u_{x x x}\right),
$$

or, applying $A$ to both sides, equivalently

$$
\mu\left(u_{t}\right)-u_{t x x}=-2 \mu(u) u_{x}+2 u_{x} u_{x x}+u u_{x x x} .
$$

Integrating both sides of this equation over the circle and using periodicity implies that $\mu\left(u_{t}\right)=0$, thus yielding the $\mu \mathrm{HS}$ equation.

An alternative derivation proceeds by calculating the first variation of the energy functional of the right-invariant metric (2.2)

$$
E(\xi)=\frac{1}{2} \int_{0}^{1}\langle\dot{\xi}(t), \dot{\xi}(t)\rangle d t
$$

defined on the space of smooth paths $t \rightarrow \xi(t)$ between $\xi(0)=$ id and $\xi(1)$ in the diffeomorphism group. The resulting geodesic equation in $\mathcal{D}^{s}\left(S^{1}\right)$ right-translated to the tangent space at the identity is the $\mu \mathrm{HS}$ equation.

Combining Theorem 2.1 with a local well-posedness result of Section 5 we will show that the metric (2.2) has a well-defined Riemannian exponential map on $\mathcal{D}^{s}\left(S^{1}\right)$ whenever $s>3 / 2$ (Corollary [5.3). 
2.2. $\mu \mathbf{H S}$ as an evolution equation. Recall that the periodic Hunter-Saxton equation

$$
u_{t x x}+2 u_{x} u_{x x}+u u_{x x x}=0
$$

is defined only as an evolution equation on the quotient $\mathcal{D}^{s}\left(S^{1}\right) / S^{1}$, i.e. on the space of cosets $\left\{u(x)+c \mid x \in S^{1}, c \in \mathbb{R}\right\}$, see e.g. [KM]. The ambiguity introduced by arbitrariness of $c$ disappears in the case of the $\mu \mathrm{HS}$ equation. Indeed, integrating equation $(\mu \mathrm{HS})$ in $x$ gives

$$
u_{t x}=2 \mu(u) u-u u_{x x}-\frac{1}{2} u_{x}^{2}+r_{0}(t)
$$

where $r_{0}(t)$ is uniquely defined at each time $t$ by the condition that the right-hand side be a total $x$-derivative. Explicitly, one has $r_{0}(t)=-2 \mu(u)^{2}-\frac{1}{2} \mu\left(u_{x}^{2}\right)$. Note that $r_{0}(t)=r_{0}$ is constant in time, since $-2 r_{0}(t)=4 \mu(u)^{2}+\mu\left(u_{x}^{2}\right)=3 \mu(u)^{2}+\langle u, u\rangle$, while both the $\mu$-value and the energy are conserved along $\mu \mathrm{HS}$ solutions. Integrating once again over $[0, x]$ gives

$$
u_{t}(t, x)=\int_{0}^{x}\left(2 \mu(u) u-u u_{x x}-\frac{1}{2} u_{x}^{2}\right) d x+r_{0} x+q_{0}(t) .
$$

The same procedure in the case of the HS equation leads to the arbitrary integration constant $q_{0}(t)$, which is the reason for HS being defined only on cosets. This time, the condition $\mu\left(u_{t}\right)=0$ implies that the mean value of the expression on the right-hand side above must be zero. This defines $q_{0}(t)$ uniquely and hence gives a well-defined evolution on the entire space of periodic functions $u: S^{1} \rightarrow \mathbb{R}$.

\section{VARIATIONAL PRINCIPLES AND PHYSICAL MOTIVATION}

\section{1. $\mu \mathrm{HS}$ as a bi-variational equation.}

Theorem 3.1. The $\mu H S$ equation satisfies two different variational principles.

We would like to show that $(\mu \mathrm{HS})$ arises as the equation $\delta S=0$ for the action functional

$$
\mathcal{S}=\int\left(\int \mathcal{L} d x\right) d t
$$

with two different densities $\mathcal{L}$.

Consider the first Lagrangian density

$$
\mathcal{L}=u_{x} u_{t}+u u_{x}^{2}+2 \mu(u) u^{2} .
$$

This is a generalization of the HS Lagrangian density where the $\mu$-term is added. The variational principle $\delta \mathcal{S}=0$ gives the Euler-Lagrange equation

$$
u_{t x}=-\frac{1}{2} u_{x}^{2}-u u_{x x}+2 \mu(u) u+\mu\left(u^{2}\right) .
$$

The right-hand-side of this equation has to be understood up to addition of a (possibly time-dependent) constant to make it a total derivative:

$$
u_{t x}=-\frac{1}{2} u_{x}^{2}-u u_{x x}+2 \mu(u) u+\mu\left(u^{2}\right)+c .
$$

Here $c$ is defined uniquely by the condition

$$
\int u_{x}^{2} d x+4 \mu(u)^{2}+2 \mu\left(u^{2}\right)+2 c=0,
$$

vanishing of the mean of the right-hand-side in (3.3). This immediately leads to equation (2.5). Equivalently, by taking the $x$-derivative, equation (3.2) can be directly understood as the $\mu$ HS equation for $u_{t x x}$. 
Remark 3.2. The additional constant term $c$ in equation (3.3) is necessitated by the term $u_{x} u_{t}$ in the Lagrangian. Indeed, consider any variational principle

$$
\delta\left(\iint u_{x} u_{t} d x d t+f(u)\right)=0 .
$$

It gives the equation $u_{t x}=-\frac{1}{2} \delta f / \delta u$, which makes sense on the circle only if the mean of the function $\delta f / \delta u$ is zero. Thus by including the term $u_{x} u_{t}$ in the Lagrangian, we are assuming that the Euler-Lagrange equation will describe the evolution of the complete derivative $u_{x}$ in the form

$$
u_{t x}=X(u),
$$

for some vector field $X$. By adding an appropriate $c$ for each moment of time to the right-hand-side, one can force the result to be a derivative.

For the Lagrangian (3.1) this constant will automatically give the appropriate value of $r_{0}$ already discussed in Section 2.2. Most importantly, this constant is non-essential and will disappear once we differentiate both sides to obtain the equation for $u_{t x x}$, as in the original $\mu \mathrm{HS}$ equation 5

The second variational representation can be obtained from the Lagrangian density

$$
\overline{\mathcal{L}}=\frac{1}{2}\left(u_{x}^{2}+\mu(u) u\right)+\pi\left(z_{t}+u z_{x}\right),
$$

which is a $\mu$-generalization of the density presented in [HZ]. Varying the corresponding action with respect to $u, \pi$, respectively $z$, we find

$$
\begin{aligned}
-u_{x x}+\mu(u)+\pi z_{x} & =0, \\
z_{t}+u z_{x} & =0, \\
-\pi_{t}-(\pi u)_{x} & =0 .
\end{aligned}
$$

Thus, using (3.5), the expression $u_{t x x}+2 u_{x} u_{x x}+u u_{x x x}$ equals

$$
\pi_{t} z_{x}+\pi z_{t x}+2 u_{x}\left(\mu(u)+\pi z_{x}\right)+u\left(\pi z_{x}\right)_{x} .
$$

After employing (3.6) and (3.7) to replace $z_{t x}$ respectively $\pi_{t}$, this simplifies to just $2 \mu(u) u_{x}$, showing that the equations of motion is the $\mu \mathrm{HS}$ equation.

As we will see in Section 4.2, these bivariational principles correspond to the two Hamiltonian formulations of the $\mu \mathrm{HS}$ equation.

3.2. Physical motivation. First recall the derivation of the HS equation from [HS]. One considers the director field $\mathbf{n}(x, y, z, t)$ which describes the average orientation of the long rod-like molecules in a nematic liquid crystal. Assuming invariance under spatial translations and no fluid flow, the action is given by (see (A.14) in [HS])

$$
\mathcal{S}=\iint\left\{\frac{1}{2}\left|\mathbf{n}_{t}\right|^{2}-W(\mathbf{n}, \nabla \mathbf{n})+\frac{1}{2} \lambda|\mathbf{n}|^{2}\right\} d \mathbf{x} d t
$$

where $\lambda$ is a Lagrangian multiplier enforcing $|\mathbf{n}|=1$, and the Oseen-Frank internal energy $W$ is

$$
W(\mathbf{n}, \nabla \mathbf{n})=\frac{1}{2}\left(k_{1}(\nabla \cdot \mathbf{n})^{2}+k_{2}(\mathbf{n} \cdot \nabla \times \mathbf{n})^{2}+k_{3}|\mathbf{n} \times(\nabla \times \mathbf{n})|^{2}\right),
$$

\footnotetext{
${ }^{5}$ It is worth mentioning that the same kind of modification by a constant is required in the variational principle for the original HS equation in the periodic case, even without the $\mu$-term.
} 
where $k_{1}, k_{2}$, and $k_{3}$, are nonnegative physical parameters describing how the material responds to splay, twist, and bend, respectively. For the director field

$$
\mathbf{n}(x, y, z, t)=\cos \psi(t, x) \mathbf{e}_{x}+\sin \psi(t, x) \mathbf{e}_{y},
$$

we get

Writing

$$
W(\mathbf{n}, \nabla \mathbf{n})=\frac{1}{2}\left(k_{1} \sin ^{2} \psi+k_{3} \cos ^{2} \psi\right) \psi_{x}^{2} .
$$

it follows that

$$
c^{2}(\psi)=k_{1} \sin ^{2} \psi+k_{3} \cos ^{2} \psi,
$$

$$
W(\mathbf{n}, \nabla \mathbf{n})=\frac{1}{2} c^{2}(\psi) \psi_{x}^{2} .
$$

Hence, since $\left|\mathbf{n}_{t}\right|^{2}=\psi_{t}^{2}$, this leads to the action

$$
\mathcal{S}=\iint \frac{1}{2}\left(\psi_{t}^{2}-c^{2}(\psi) \psi_{x}^{2}\right) d x d t .
$$

(Note that the Lagrange multiplier $\lambda$ does not appear because $\mathbf{n}$ is already normalized to have length one.) The Euler-Lagrange equation, obtained in [HS] for the action $\mathcal{S}$ is

$$
\psi_{t t}-c(\psi)\left[c(\psi) \psi_{x}\right]_{x}=0 .
$$

At this point we deviate from the HS derivation and assume that there is a preferred direction $\psi_{0}$ of the director field. This can be related, e.g., to an exterior magnetic field, acting on dipoles and returning them to this direction. Such a returning force corresponds to the addition of the potential energy term $-\frac{k}{2}\left(\psi-\psi_{0}\right)^{2}$ to the Lagrangian density $\mathcal{L}=\frac{1}{2}\left(\psi_{t}^{2}-c^{2}(\psi) \psi_{x}^{2}\right)$. We also assume that there is retroactive action of other dipoles on a given director, i.e. that Hooke's elasticity coefficient $k:=\mu\left(\psi-\psi_{0}\right)$ is proportional to the average deviation of all dipoles from $\psi_{0}$ : other dipoles act on a given one, and the more other dipoles are rotated, the stronger the returning force is on the given dipole.

Thus the new action is

$$
\mathcal{S}=\frac{1}{2} \iint\left(\psi_{t}^{2}-c^{2}(\psi) \psi_{x}^{2}-\mu\left(\psi-\psi_{0}\right)\left(\psi-\psi_{0}\right)^{2}\right) d x d t
$$

where $\psi_{0}$ is the direction about which the fluctuations occur 6 We need to choose $\psi_{0}$ so that both $c_{0}:=c\left(\psi_{0}\right)$, as well as $c_{0}^{\prime}:=c^{\prime}\left(\psi_{0}\right)$, are nonzero.

The Euler-Lagrange equation now becomes

$$
\psi_{t t}-c(\psi)\left[c(\psi) \psi_{x}\right]_{x}+\mu\left(\psi-\psi_{0}\right)\left(\psi-\psi_{0}\right)+\frac{1}{2} \mu\left(\left(\psi-\psi_{0}\right)^{2}\right)=0 .
$$

Now, repeating the derivation in $[\mathrm{HS}]$ with this modified equation, we introduce the variables

$$
\theta=x-c_{0} t, \quad \tau=\epsilon t,
$$

and substitute the expansions

$$
\psi=\psi_{0}+\epsilon \psi_{1}(\theta, \tau)+\epsilon^{2} \psi_{2}(\theta, \tau)+O\left(\epsilon^{3}\right) \quad \text { and } \quad c(\psi)=c_{0}+\epsilon c_{0}^{\prime} \psi_{1}+O\left(\epsilon^{2}\right)
$$

into (3.8). One finds

$$
\left(-c_{0} \partial_{\theta}+\epsilon \partial_{\tau}\right)^{2}\left(\epsilon \psi_{1}+\epsilon^{2} \psi_{2}\right)-\left(c_{0}+\epsilon c_{0}^{\prime} \psi_{1}\right)\left[\left(c_{0}+\epsilon c_{0}^{\prime} \psi_{1}\right)\left(\epsilon \psi_{1 \theta}+\epsilon^{2} \psi_{2 \theta}\right)\right]_{\theta}
$$

\footnotetext{
${ }^{6}$ The added term does not preserve the periodicity of the angle $\psi$, but this is inessential since when deriving the asymptotic equation we only consider small fluctuations of $\psi$.
} 


$$
+\left(\epsilon \psi_{1}+\epsilon^{2} \psi_{2}\right) \int\left(\epsilon \psi_{1}+\epsilon^{2} \psi_{2}\right) d x+\frac{1}{2} \int\left(\epsilon \psi_{1}+\epsilon^{2} \psi_{2}\right)^{2} d x+O\left(\epsilon^{3}\right)=0,
$$

where we used that

$$
\partial_{t}=-c_{0} \partial_{\theta}+\epsilon \partial_{\tau} \quad \text { and } \quad \partial_{x}=\partial_{\theta} .
$$

Collecting terms of different order we see that both the $O(1)$ and the $O(\epsilon)$ levels vanish, whereas the $O\left(\epsilon^{2}\right)$ terms give

$c_{0}^{2} \psi_{2 \theta \theta}-2 c_{0} \psi_{1 \theta \tau}-c_{0} c_{0}^{\prime}\left[\psi_{1} \psi_{1 \theta}\right]_{\theta}-c_{0}^{2} \psi_{2 \theta \theta}-c_{0} c_{0}^{\prime} \psi_{1} \psi_{1 \theta \theta}+\psi_{1} \int \psi_{1} d x+\frac{1}{2} \int \psi_{1}^{2} d x=0$.

This is simplified to

$$
\psi_{1 \theta \tau}=-\frac{c_{0}^{\prime}}{2}\left(\psi_{1 \theta}^{2}+2 \psi_{1} \psi_{1 \theta \theta}\right)+\frac{\psi_{1}}{2 c_{0}} \int \psi_{1} d x+\frac{1}{4 c_{0}} \int \psi_{1}^{2} d x .
$$

Replacing $\tau \rightarrow t, \theta \rightarrow x$, and $\psi_{1} \rightarrow u / c_{0}^{\prime}$, we arrive at

$$
u_{t x}=-\frac{1}{2} u_{x}^{2}-u u_{x x}+\frac{1}{2 c_{0} c_{0}^{\prime}}\left(u \mu(u)+\frac{1}{2} \mu\left(u^{2}\right)\right) .
$$

This is the $\mu \mathrm{HS}$ equation in the form (3.2) when $\frac{1}{2 c_{0} c_{0}^{\prime}}=2$. After differentiation this leads to the evolution equation ( $\mu \mathrm{HS}$ ) for $u_{t x x}$.

\section{HAMiltonian FRAMEWORK AND CONSERVATION LAWS}

\subsection{Bihamiltonian structure and the $\mu \mathrm{HS}$ hierarchy.}

Theorem 4.1. The $\mu H S$ equation is Hamiltonian with respect to two compatible Hamiltonian structures.

Proof Let $m:=A u=\mu(u)-u_{x x}$ and define

$$
H_{1}=\frac{1}{2} \int u m d x=\frac{1}{2}\langle u, u\rangle, \quad H_{2}=\int\left(\mu(u) u^{2}+\frac{1}{2} u u_{x}^{2}\right) d x .
$$

Then

$$
\frac{\delta H_{1}}{\delta u}=m, \quad \frac{\delta H_{2}}{\delta u}=\mu\left(u^{2}\right)+2 \mu(u) u-\frac{1}{2} u_{x}^{2}-u u_{x x} .
$$

Since $A$ is self-adjoint, we have the relation $\frac{\delta H_{1}}{\delta u}=A \frac{\delta H_{1}}{\delta m}$. Hence

$$
\frac{\delta H_{1}}{\delta m}=u, \quad \frac{\delta H_{2}}{\delta m}=A^{-1}\left(\mu\left(u^{2}\right)+2 \mu(u) u-\frac{1}{2} u_{x}^{2}-u u_{x x}\right),
$$

so that the $\mu \mathrm{HS}$ equation

$$
-u_{t x x}=-2 \mu(u) u_{x}+2 u_{x} u_{x x}+u u_{x x x},
$$

can be written both as

and as

$$
m_{t}=\mathcal{B}_{1} \frac{\delta H_{1}}{\delta m}
$$

$$
m_{t}=\mathcal{B}_{2} \frac{\delta H_{2}}{\delta m}
$$

where the Hamiltonian operators are

$$
\mathcal{B}_{1}=-\left(m \partial_{x}+\partial_{x} m\right), \quad \mathcal{B}_{2}=-\partial_{x} A=\partial_{x}^{3} .
$$

These operators are compatible as a straightforward but lengthy calculation shows. We will present their geometric meaning in the more general setting of the Virasoro algebra in Section 8 , 
Using this bihamiltonian structure it is well-known that one can (at least formally) obtain an infinite hierarchy of conservation laws, cf. McK2. If two functionals $F_{0}$ and $F_{1}$ satisfy

$$
\mathcal{B}_{1} \frac{\delta F_{0}}{\delta m}=\mathcal{B}_{2} \frac{\delta F_{1}}{\delta m}
$$

we say that $F_{0}$ raises to $F_{1}$ or that $F_{1}$ lowers to $F_{0}$; in symbols $F_{0} \nearrow F_{1}$ or $F_{1} \searrow F_{0}$. Starting from $H_{1}$ and $H_{2}$ the goal is to construct an infinite sequence of conservation laws such that

$$
\cdots \nearrow H_{-1} \nearrow H_{0} \nearrow H_{1} \nearrow H_{2} \nearrow H_{3} \nearrow \cdots .
$$

Just like for the Camassa-Holm equation, we find that lowering of the $H_{n}$ 's is unobstructed for the $\mu \mathrm{HS}$ equation, i.e. there are $H_{-1}, H_{-2}, \ldots$, such that $H_{-1} \searrow$ $H_{-2} \searrow \ldots$ Moreover, $H_{-1}, H_{-2}, \ldots$ are local functionals given by

$$
H_{-n}=\frac{1}{3 / 2-n} \int m \frac{\delta H_{-n}}{\delta m} d x, \quad n=1,2, \ldots,
$$

where $\frac{\delta H_{-n-1}}{\delta m}$ is obtained from $\frac{\delta H_{-n}}{\delta m}$ via

$$
\frac{\delta H_{-n-1}}{\delta m}=\mathcal{B}_{1}^{-1} \mathcal{B}_{2} \frac{\delta H_{-n}}{\delta m}=-\frac{1}{2 \sqrt{m}} \int^{x} \frac{1}{\sqrt{m}}\left(\frac{\delta H_{-n}}{\delta m}\right)_{x x x} d x .
$$

Starting with $\delta H_{-1}=\frac{1}{2 \sqrt{m}}$ this provides a constructive approach for finding the $H_{-n}$ 's, which is easily implemented in Mathematica. For example, we get

$$
\begin{gathered}
\frac{\delta H_{-2}}{\delta m}=\frac{m_{x x}}{8 m^{5 / 2}}-\frac{5 m_{x}^{2}}{32 m^{7 / 2}}, \\
\frac{\delta H_{-3}}{\delta m}=\frac{1155 m_{x}^{4}}{1024 m^{13 / 2}}-\frac{231 m_{x}^{2} m_{x x}}{128 m^{11 / 2}}+\frac{21 m_{x x}^{2}}{64 m^{9 / 2}}+\frac{7 m_{x} m_{x x x}}{16 m^{9 / 2}}-\frac{m_{x x x x}}{16 m^{7 / 2}},
\end{gathered}
$$

and the corresponding functionals $H_{-2}$ and $H_{-3}$ are obtained from (4.2).

The situation for raising is more intricate due to the occurence of nonlocal functionals. Nevertheless, we can deduce the existence of expressions $Q_{n}[m]$ (possibly nonlocal in $m$ ) such that $Q_{0}=\frac{\delta H_{0}}{\delta m}$ and

$$
Q_{n+1}=\mathcal{B}_{2}^{-1} \mathcal{B}_{1} Q_{n}, \quad n \geq 0 .
$$

If there exist functionals $H_{n}[m]$ with $\frac{\delta H_{n}}{\delta m}=Q_{n}$, they constitute an infinite ladder of functionals with $H_{1} \nearrow H_{2} \nearrow H_{2} \nearrow \cdots$. The existence of the $H_{n}$ is not evident in this approach due to the nonlocal nature of the $Q_{n}$ 's. However, it follows from the expansion of a Casimir for a family of compatible Poisson brackets in the approach of Section 8, cf. [KM].

In summary, the first few conservation laws in the hierarchy are

$$
\begin{aligned}
H_{-2}=-\frac{1}{16} \int \frac{m_{x}^{2}}{m^{5 / 2}} d x, & \frac{\delta H_{-2}}{\delta m}=\frac{m_{x x}}{8 m^{5 / 2}}-\frac{5 m_{x}^{2}}{32 m^{7 / 2}}, \\
H_{-1}=\int \sqrt{m} d x, & \frac{\delta H_{-1}}{\delta m}=\frac{1}{2 \sqrt{m}}, \\
H_{0}=\int m d x, & \frac{\delta H_{0}}{\delta m}=1, \\
H_{1}=\frac{1}{2} \int m u d x, & \frac{\delta H_{1}}{\delta m}=u, \\
H_{2}=\int\left(\mu(u) u^{2}+\frac{1}{2} u u_{x}^{2}\right) d x, & \frac{\delta H_{2}}{\delta m}=\left(\mu-\partial_{x}^{2}\right)^{-1}\left(\mu\left(u^{2}\right)+2 \mu(u) u-\frac{1}{2} u_{x}^{2}-u u_{x x}\right) .
\end{aligned}
$$


with corresponding commuting flows

$$
\begin{aligned}
& m_{t}=\mathcal{B}_{1} \frac{\delta H_{-2}}{\delta m}=\mathcal{B}_{2} \frac{\delta H_{-1}}{\delta m}=\left(\frac{1}{2 \sqrt{m}}\right)_{x x x}, \\
& m_{t}=\mathcal{B}_{1} \frac{\delta H_{-1}}{\delta m}=\mathcal{B}_{2} \frac{\delta H_{0}}{\delta m}=0, \\
& m_{t}=\mathcal{B}_{1} \frac{\delta H_{0}}{\delta m}=\mathcal{B}_{2} \frac{\delta H_{1}}{\delta m}=-m_{x}, \\
& m_{t}=\mathcal{B}_{1} \frac{\delta H_{1}}{\delta m}=\mathcal{B}_{2} \frac{\delta H_{2}}{\delta m}=-2 m u_{x}-m_{x} u .
\end{aligned}
$$

4.2. Relation with bi-variational principles. Recall that the $\mu$ HS equation can be obtained from two distinct variational principles, corresponding to the Lagrangian densities $\mathcal{L}$ and $\overline{\mathcal{L}}$ introduced in Section 3 .

Theorem 4.2. The two variational formulations for the $\mu H S$ equation formally correspond to the two Hamiltonian formulations of this equation with Hamiltonian functionals $H_{1}$ and $H_{2}$.

Proof The action is related to the Lagrangian by $S=\int L d t$ and $L=\int \mathcal{L} d x$. The first variational principle has the Lagrangian density of equation (3.1),

$$
\mathcal{L}=u_{x} u_{t}+u u_{x}^{2}+2 \mu(u) u^{2}
$$

where we skip the linear term. The momenta conjugate to the velocities $u_{t}$ are

$$
\frac{\partial \mathcal{L}}{\partial u_{t}}=u_{x}
$$

Hence the Hamiltonian density is

$$
\mathcal{H}=u_{x} u_{t}-\mathcal{L}=-u u_{x}^{2}-2 \mu(u) u^{2},
$$

and so

$$
H=-\int\left(u u_{x}^{2}+2 \mu(u) u^{2}\right) d x,
$$

which indeed agress with the conservation law $\mathrm{H}_{2}$ up to a factor of -2 .

In the second principle the Lagrangian density is

$$
\overline{\mathcal{L}}=\frac{1}{2} u_{x}^{2}+\frac{1}{2} \mu(u) u+\pi\left(z_{t}+u z_{x}\right) .
$$

The momenta conjugate to the velocities $u_{t}, \pi_{t}$, and $z_{t}$, respectively, are

$$
\frac{\partial \overline{\mathcal{L}}}{\partial u_{t}}=0, \quad \frac{\partial \overline{\mathcal{L}}}{\partial \pi_{t}}=0, \quad \frac{\partial \overline{\mathcal{L}}}{\partial z_{t}}=\pi .
$$

Consequently, the Hamiltonian density is

$$
\mathcal{H}=z_{t} \pi-\overline{\mathcal{L}}=z_{t} \pi-\left(\frac{1}{2} u_{x}^{2}+\frac{1}{2} \mu(u) u+\pi\left(z_{t}+u z_{x}\right)\right) .
$$

Using the equation of motion (3.5) to substitute for $\pi z_{x}$, this becomes

$$
\mathcal{H}=-\frac{1}{2} u_{x}^{2}-u u_{x x}+\frac{1}{2} \mu(u) u .
$$

Therefore, the Hamiltonian is

$$
H=\int \mathcal{H} d x=\frac{1}{2} \int\left(u_{x}^{2}+\mu(u) u\right) d x
$$

which is exactly $H_{1}$. 
Remark 4.3. (Lax Pair) The $\mu \mathrm{HS}$ equation

$$
u_{t x x}-2 \mu(u) u_{x}+2 u_{x} u_{x x}+u u_{x x x}=0 .
$$

can be viewed as the condition of compatibility between

$$
\psi_{x x}=\lambda m \psi
$$

and

$$
\psi_{t}=\left(\frac{1}{2 \lambda}-u\right) \psi_{x}+\frac{1}{2} u_{x} \psi
$$

where $\lambda$ is a spectral parameter and, as above, $m=\mu(u)-u_{x x}$. In other words, assuming that these equations are satisfied for all nonzero $\lambda \in \mathbb{C}$, the relation $\psi_{t x x}=$ $\psi_{x x t}$ holds if and only if $u(t, x)$ satisfies the $\mu \mathrm{HS}$ equation.

\section{The PeRiodic CAuChy PROBlem}

In this section we discuss well-posedness of the $\mu \mathrm{HS}$ equation. We do not aim for the strongest possible results here. Instead, after establishing a basic local wellposedness theorem we proceed to show that while some solutions persist indefinitely other solutions develop singularities in finite time.

Recall that the mean of any solution $u=u(t, x)$ is conserved by the flow and hence the Cauchy problem for the $\mu \mathrm{HS}$ equation can be recast in the form

$$
u_{t}+u u_{x}+A^{-1} \partial_{x}\left(2 \mu(u) u+\frac{1}{2} u_{x}^{2}\right)=0
$$

and

$$
u(0, x)=u_{o}(x), \quad x \in S^{1}, \quad t \in \mathbb{R},
$$

where $A=\mu-\partial_{x}^{2}$ is an isomorphism between $H^{s}\left(S^{1}\right)$ and $H^{s-2}\left(S^{1}\right)$ with inverse $u=A^{-1} w$ given explicitly by equation (2.3). Conservation of the mean will play an important role later on.

5.1. Local well-posedness and persistence result. In order to establish local well-posedness of the Cauchy problem (5.1) - (5.2) we adopt here the approach developed in [M2] for the $\mathrm{CH}$ equation. Our main result is the following

Theorem 5.1. If $s>3 / 2$ then for any $u_{o} \in H^{s}\left(S^{1}\right)$ there exist $T>0$ and a unique solution $u \in C\left((-T, T), H^{s}\right) \cap C\left((-T, T), H^{s-1}\right)$ of the initial value problem (5.1)(5.2) which depends continuously on the initial data $u_{o}$. Furthermore, the solution persists as long as $\|u(t, \cdot)\|_{C^{1}}$ stays bounded.

First, however, we will study the associated Cauchy problem on the group $\mathcal{D}^{s}\left(S^{1}\right)$ of Sobolev $H^{s}$ diffeomorphisms with $s>3 / 2$. Given a solution $u(t, x)$ of (5.1) with initial data $u_{o}$ we let $t \rightarrow \eta(t, x)$ be the flow of $u$, that is

$$
\dot{\eta}(t, x)=u(t, \eta(t, x)) .
$$

Differentiating both sides of (5.3) with respect to $t$ we rewrite the original Cauchy problem (5.1) -(5.2) as

$$
\begin{gathered}
\ddot{\eta}=-\left\{A^{-1} \partial_{x}\left(2 \mu\left(\dot{\eta} \circ \eta^{-1}\right) \dot{\eta} \circ \eta^{-1}+\frac{1}{2}\left(\left(\dot{\eta} \circ \eta^{-1}\right)_{x}\right)^{2}\right)\right\} \circ \eta=: f(\eta, \dot{\eta}) \\
\eta(0, x)=x, \quad \dot{\eta}(0, x)=u_{o}(x)
\end{gathered}
$$

and prove 
Theorem 5.2. Let $s>3 / 2$. For any $u_{o} \in H^{s}\left(S^{1}\right)$ there exist $T>0$ and a unique solution $(\eta, \dot{\eta}) \in C^{1}\left((-T, T), \mathcal{D}^{s}\left(S^{1}\right) \times H^{s}\right)$ of the initial value problem (5.4)-(5.5) which depends differentiably on the initial data $u_{o}$.

An immediate consequence of Theorem 5.2 combined with the inverse function theorem for Banach spaces is the existence of the Riemannian exponential map in $\mathcal{D}^{s}\left(S^{1}\right)$ whose geodesics were described in Theorem 2.1. The corollary below permits a rigorous development of the associated infinite-dimensional Riemannian geometry and justifies the geometric constructions of Sections 2 and 7.

Corollary 5.3. For any $s>3 / 2$ the metric (2.2) has a well-defined exponential map $\exp _{\mathrm{id}}: U \subset T_{\mathrm{id}} \mathcal{D}^{s}\left(S^{1}\right) \rightarrow \mathcal{D}^{s}\left(S^{1}\right)$ which is a $C^{1}$ diffeomorphism from an open set $U$ onto a neighbourhood of the identity in $\mathcal{D}^{s}\left(S^{1}\right)$.

Our first main task will be to show that the map $(\xi, w) \rightarrow(w, f(\xi, w))$ defines a $C^{1}$ vector field on the Hilbert manifold $\mathcal{D}^{s}\left(S^{1}\right) \times H^{s}\left(S^{1}\right)$. The proof of this fact is similar to the corresponding proof for $\mathrm{CH}$ and we will refer the reader to [M2] for some of the details.

Proof of Theorem 5.2. It is sufficient to show that $f$ is continuously differentiable (in the Fréchet sense) with respect to the variables $\xi$ and $w$ in some neighbourhood of the point (id, 0$)$ in $\mathcal{D}^{s}\left(S^{1}\right) \times H^{s}\left(S^{1}\right)$. The following technical result will be useful.

Lemma 5.4. (鱼2, Appendix 1) For $s>3 / 2$ the composition map $\xi \rightarrow w \circ \xi$ with an $H^{s}$ function $w$ and the inversion map $\xi \rightarrow \xi^{-1}$ are continuous from $\mathcal{D}^{s}\left(S^{1}\right)$ to $H^{s}\left(S^{1}\right)$ and from $\mathcal{D}^{s}\left(S^{1}\right)$ to itself respectively. Moreover

$$
\|w \circ \xi\|_{H^{s}} \leq C\left(1+\|\xi\|_{H^{s}}^{s}\right)\|w\|_{H^{s}}
$$

with $C$ depending only on $\inf \left|\partial_{x} \xi\right|$ and $\sup \left|\partial_{x} \xi\right|$.

In particular, using (5.6) we find that the right hand side of (5.4) maps $(\xi, w) \rightarrow$ $f(\xi, w)$ into $H^{s}\left(S^{1}\right)$.

Next, rewrite $f$ in the form

$$
f(\xi, w)=-A_{\xi}^{-1} \partial_{x, \xi} h(\xi, w),
$$

where $\partial_{x, \xi}=d R_{\xi} \circ \partial_{x} \circ d R_{\xi^{-1}}$ and $A_{\xi}^{-1}=d R_{\xi} \circ A^{-1} \circ d R_{\xi^{-1}}$ are the conjugations of $\partial_{x}$ and $A^{-1}$ by a diffeomorphism $\xi$ (here $R_{\xi}$ denotes the right translation by $\xi$ in $\left.\mathcal{D}^{s}\left(S^{1}\right)\right)$ and where

$$
h(\xi, w)=2 w \int w \circ \xi^{-1} d x+\frac{1}{2}\left(\frac{\partial_{x} w}{\partial_{x} \xi}\right)^{2} .
$$

Let $s \rightarrow \xi_{s}$ be a smooth curve in $\mathcal{D}^{s}\left(S^{1}\right)$ passing through the point $\xi_{0}=\xi$ in the direction $\left.\partial_{s} \xi_{s}\right|_{s=0}=v$. A formal computation gives

$$
\partial_{\xi}\left(\partial_{x, \xi}\right)(v)=\left.\partial_{s}\left(\partial_{x, \xi_{s}}\right)\right|_{s=0}=\left[v \circ \xi^{-1} \partial_{x}, \partial_{x}\right]_{\xi}
$$

and

$$
\partial_{\xi}\left(A_{\xi}^{-1}\right)(v)=\left.\partial_{s}\left(A_{\xi_{s}}^{-1}\right)\right|_{s=0}=-\left.A_{\xi}^{-1} \partial_{s}\left(A_{\xi_{s}}\right)\right|_{s=0} A_{\xi}^{-1}=-A_{\xi}^{-1}\left[v \circ \xi^{-1} \partial_{x}, A\right]_{\xi} A_{\xi}^{-1},
$$

where $[\cdot, \cdot]$ denotes the commutator of operators. Similarly from (5.8) we find

$$
\partial_{\xi} h_{(\xi, w)}(v)=2\left\{w \circ \xi^{-1} \int w \circ \xi^{-1} \partial_{x}\left(v \circ \xi^{-1}\right) d x-\frac{1}{2}\left(\partial_{x}\left(w \circ \xi^{-1}\right)\right)^{2} \partial_{x}\left(v \circ \xi^{-1}\right)\right\} \circ \xi,
$$


as well as

$$
\begin{aligned}
\partial_{w} h_{(\xi, w)}(v)=2\left\{w \circ \xi^{-1} \int v \circ \xi^{-1} d x\right. & +v \circ \xi^{-1} \int w \circ \xi^{-1} d x \\
& \left.+\frac{1}{2} \partial_{x}\left(w \circ \xi^{-1}\right) \partial_{x}\left(v \circ \xi^{-1}\right)\right\} \circ \xi .
\end{aligned}
$$

With the help of the above formulas we can compute the directional derivatives of $f$ in (5.7), namely

$$
\begin{aligned}
& \partial_{\xi} f_{(\xi, w)}(v)=-\partial_{\xi}\left(A_{\xi}^{-1}\right)(v) \partial_{x, \xi} h(\xi, w)-A_{\xi}^{-1} \partial_{\xi}\left(\partial_{x, \xi}\right)(v) h(\xi, w)-A_{\xi}^{-1} \partial_{x, \xi} \partial_{\xi} h_{(\xi, w)}(v) \\
&=\left\{A^{-1} \partial_{x}\left(v \circ \xi^{-1} \partial_{x}\left(h \circ \xi^{-1}\right)\right)-v \circ \xi^{-1} \partial_{x} A^{-1} \partial_{x}\left(h \circ \xi^{-1}\right)\right\} \circ \xi \\
&-2\left\{A^{-1} \partial_{x}\left(w \circ \xi^{-1} \int w \circ \xi^{-1} \partial_{x}\left(v \circ \xi^{-1}\right) d x-\frac{1}{2}\left(\partial_{x}\left(w \circ \xi^{-1}\right)\right)^{2} \partial_{x}\left(v \circ \xi^{-1}\right)\right)\right\} \circ \xi
\end{aligned}
$$

and

$$
\begin{aligned}
\partial_{w} f_{(\xi, w)}(v) & =-\left(A^{-1} \partial_{x}\right)_{\xi} \partial_{w} h_{(\xi, w)}(v) \\
=-2\left\{A ^ { - 1 } \partial _ { x } \left(w \circ \xi^{-1} \int v \circ \xi^{-1} d x\right.\right. & +v \circ \xi^{-1} \int w \circ \xi^{-1} d x \\
& \left.\left.+\frac{1}{2} \partial_{x}\left(w \circ \xi^{-1}\right) \partial_{x}\left(v \circ \xi^{-1}\right)\right)\right\} \circ \xi .
\end{aligned}
$$

Our strategy will be to show that both directional derivatives $\partial_{\xi} f_{(\xi, w)}$ and $\partial_{w} f_{(\xi, w)}$ are bounded operators in $L\left(H^{s}, H^{s}\right)$ that depend continuously on $(\xi, w)$ in some neighbourhood of (id, 0$)$. Using the identities

$$
A^{-1} \partial_{x} w(x)=\left(x-\frac{1}{2}\right) \int w(x) d x-\int_{0}^{x} w(y) d y+\iint_{0}^{x} w(y) d y d x
$$

and

$$
A^{-1} \partial_{x}^{2} w(x)=-w(x)+\int w(x) d x
$$

which are easily obtained from (2.3) and the fact that $A^{-1}$ and $\partial_{x}$ commute, we find explicit expressions for the directional derivatives in (5.11) and (5.12). After a 
lengthy, but straightforward, calculation we obtain

$$
\begin{aligned}
& \partial_{\xi} f_{(\xi, w)}(v)\left\{4\left(x-\frac{1}{2}\right) \int w \circ \xi^{-1} d x \int v \circ \xi^{-1} \partial_{x}\left(w \circ \xi^{-1}\right) d x\right. \\
&+ \frac{1}{2}\left(x-\frac{1}{2}\right) \int\left(\partial_{x}\left(w \circ \xi^{-1}\right)\right)^{2} \partial_{x}\left(v \circ \xi^{-1}\right) d x \\
&+2 \int w \circ \xi^{-1} \partial_{x}\left(v \circ \xi^{-1}\right) d x \int_{0}^{x} w \circ \xi^{-1} d y-2 \int w \circ \xi^{-1} d x \int_{0}^{x} v \circ \xi^{-1} \partial_{x}\left(w \circ \xi^{-1}\right) d y \\
&-\frac{1}{2} \int_{0}^{x}\left(\partial_{x}\left(w \circ \xi^{-1}\right)\right)^{2} \partial_{x}\left(v \circ \xi^{-1}\right) d y \\
&+ 2 \int w \circ \xi^{-1} d x \iint_{0}^{x} v \circ \xi^{-1} \partial_{x}\left(w \circ \xi^{-1}\right) d y d x \\
&-2 \int w \circ \xi^{-1} \partial_{x}\left(v \circ \xi^{-1}\right) d x \iint_{0}^{x} w \circ \xi^{-1} d y d x \\
&+\frac{1}{2} \int v \circ \xi^{-1}\left(\partial_{x}\left(w \circ \xi^{-1}\right)\right)^{2} d x+\frac{1}{2} \iint_{0}^{x}\left(\partial_{x}\left(w \circ \xi^{-1}\right)\right)^{2} \partial_{x}\left(v \circ \xi^{-1}\right) d y d x \\
&+2 v \circ \xi^{-1} w \circ \xi^{-1} \int w \circ \xi^{-1} d x-2 v \circ \xi^{-1}\left(\int w \circ \xi^{-1} d x\right)^{2} \\
&\left.-\frac{1}{2} v \circ \xi^{-1} \int\left(\partial_{x}\left(w \circ \xi^{-1}\right)\right)^{2} d x\right\} \circ \xi
\end{aligned}
$$

and similarly

$$
\begin{aligned}
& \partial_{w} f_{(\xi, w)}(v)=-\left\{4\left(x-\frac{1}{2}\right) \int w \circ \xi^{-1} d x \int v \circ \xi^{-1} d x\right. \\
&+\left(x-\frac{1}{2}\right) \int \partial_{x}\left(w \circ \xi^{-1}\right) \partial_{x}\left(v \circ \xi^{-1}\right) d x-2 \int w \circ \xi^{-1} d x \int_{0}^{x} v \circ \xi^{-1} d y \\
&-2 \int v \circ \xi^{-1} d x \int_{0}^{x} w \circ \xi^{-1} d y-\int_{0}^{x} \partial_{x}\left(w \circ \xi^{-1}\right) \partial_{x}\left(v \circ \xi^{-1}\right) d y \\
&+2 \int w \circ \xi^{-1} d x \iint_{0}^{x} v \circ \xi^{-1} d y d x+2 \int v \circ \xi^{-1} d x \iint_{0}^{x} w \circ \xi^{-1} d y d x \\
&\left.+\iint_{0}^{x} \partial_{x}\left(w \circ \xi^{-1}\right) \partial_{x}\left(v \circ \xi^{-1}\right) d y d x\right\} \circ \xi .
\end{aligned}
$$

By Lemma 5.4, in order to show that $v \rightarrow \partial_{\xi} f_{(\xi, w)}(v)$ is a bounded operator on $H^{s}$ it will be sufficient to estimate the sum

$$
\left\|\partial_{\xi} f_{(\xi, w)}(v) \circ \xi^{-1}\right\|_{L^{2}}+\left\|\partial_{x}\left(\partial_{\xi} f_{(\xi, w)}(v)\right) \circ \xi^{-1}\right\|_{H^{s-1}} .
$$

Using Cauchy-Schwarz and the Sobolev lemma we can bound the first of the two terms above by

$$
\begin{aligned}
& \lesssim\left(\left\|w \circ \xi^{-1}\right\|_{\infty}\left\|w \circ \xi^{-1}\right\|_{H^{1}}+\left\|w \circ \xi^{-1}\right\|_{H^{1}}^{2}\right)\left(\left\|v \circ \xi^{-1}\right\|_{H^{1}}+\left\|v \circ \xi^{-1}\right\|_{C^{1}}\right) \\
& \lesssim\left\|w \circ \xi^{-1}\right\|_{H^{1}}^{2}\left\|v \circ \xi^{-1}\right\|_{H^{s}}
\end{aligned}
$$


and, after differentiating (5.15) in $x$ and using the fact that $H^{s-1}$ is an algebra, estimate the second term by

$$
\begin{aligned}
& \lesssim\left\|\partial_{x}\left(w \circ \xi^{-1}\right)\right\|_{L^{2}}^{2}\left\|\partial_{x}\left(v \circ \xi^{-1}\right)\right\|_{\infty}+\left\|\partial_{x}\left(w \circ \xi^{-1}\right)\right\|_{H^{s-1}}^{2}\left\|\partial_{x}\left(v \circ \xi^{-1}\right)\right\|_{H^{s-1}} \\
& +\left\|w \circ \xi^{-1}\right\|_{\infty}\left\|w \circ \xi^{-1}\right\|_{H^{s}}\left\|v \circ \xi^{-1}\right\|_{H^{s}}+\left\|w \circ \xi^{-1}\right\|_{L^{2}}^{2}\left\|\partial_{x}\left(v \circ \xi^{-1}\right)\right\|_{H^{s-1}} \\
& \lesssim\left\|w \circ \xi^{-1}\right\|_{H^{s}}^{2}\left\|v \circ \xi^{-1}\right\|_{H^{s}}
\end{aligned}
$$

Combining these and using the estimate (5.6) of Lemma 5.4 we obtain

$$
\left\|\partial_{\xi} f_{(\xi, w)}(v)\right\|_{H^{s}} \lesssim C\|w\|_{H^{s}}^{2}\|v\|_{H^{s}},
$$

where the constant $C$ depends only on the $H^{s}$ norms of $\partial_{x} \xi$ and $\partial_{x} \xi^{-1}$. An analogous argument yields a similar bound on $\partial_{w} f_{(\xi, w)}$ in (5.16)

$$
\left\|\partial_{w} f_{(\xi, w)}(v)\right\|_{H^{s}} \lesssim C\|w\|_{H^{s}}\|v\|_{H^{s}} .
$$

We have thus shown that $f$ is Gateaux differentiable near (id, 0 ).

We next turn to continuity of the directional derivatives with respect to the variables $\xi$ and $w$. Continuity in $w$ follows from the fact that the dependence of both partials on this variable is polynomial. Therefore, it only remains to show that the norm of the difference $\left\|\partial_{\xi} f_{(\xi, w)}(v)-\partial_{\xi} f_{(\mathrm{id}, w)}(v)\right\|_{H^{s}}$ can be made arbitrarily small whenever $\xi$ is close to id in $\mathcal{D}^{s}\left(S^{1}\right)$, uniformly in $v, w \in H^{s}\left(S^{1}\right)$. Thus, our task is to estimate the sum

$$
\left\|\partial_{\xi} f_{(\xi, w)}(v)-\partial_{\xi} f_{(\mathrm{id}, w)}(v)\right\|_{L^{2}}+\left\|\partial_{x}\left(\partial_{\xi} f_{(\xi, w)}(v)-\partial_{\xi} f_{(\mathrm{id}, w)}(v)\right)\right\|_{H^{s-1}}
$$

Using the explicit formulas in (5.15) together with the estimate of Lemma 5.4, the algebra property of $H^{s-1}$ and the fact that for any $r>0$ we have a Sobolev embedding into Hölder spaces $H^{r}\left(S^{1}\right) \hookrightarrow C^{r-1 / 2}\left(S^{1}\right)$ with a pointwise estimate

$$
|g(x)-g(y)| \lesssim\|g\|_{H^{r}}|x-y|^{r-1 / 2} \quad\left(x, y \in S^{1}\right)
$$

both terms in the expression above can be bounded by

$$
\lesssim C\|w\|_{H^{s}}^{2}\|v\|_{H^{s}}\left(\left\|\xi^{-1}-\mathrm{id}\right\|_{H^{s}}+\left\|\xi^{-1}-\mathrm{id}\right\|_{\infty}^{s-3 / 2}\right),
$$

where $C$ depends only on the $H^{s}$ norms of $\xi$ and its inverse. The arguments needed to prove continuity of the map $\xi \rightarrow \partial_{w} f_{(\xi, w)}$ are very similar.

From these estimates we conclude that $f$ defined by the right side of (5.4) is continuously differentiable in a neighbourhood of the point (id,0). Applying the fundamental ODE theorem for Banach spaces we obtain Theorem 5.2 ,

We are now ready to prove local well-posedness and persistence results for the $\mu \mathrm{HS}$ equation.

Proof of Theorem 5.1. Observe that directly from the flow equation (5.3) we have $u=\dot{\eta} \circ \eta^{-1}$. Thererfore, since $\mathcal{D}^{s}\left(S^{1}\right)$ is a topological group by Lemma 5.4, the first statement is a direct corollary of Theorem 5.2 .

To prove the persistence result we employ Friedrichs' mollifiers $J_{\varepsilon} \in O P S^{-\infty}$, where $0<\varepsilon<1$, and using (5.1) we first derive an estimate for

$$
\begin{aligned}
\frac{d}{d t}\left\|J_{\varepsilon} u\right\|_{H^{s}}^{2}= & -2\left\langle\Lambda^{s} J_{\varepsilon}\left(u \partial_{x} u\right), \Lambda^{s} J_{\varepsilon} u\right\rangle_{L^{2}}-4\left\langle\Lambda^{s} A^{-1} \partial_{x} J_{\varepsilon}(\mu(u) u), \Lambda^{s} J_{\varepsilon} u\right\rangle_{L^{2}} \\
& -\left\langle\Lambda^{s} A^{-1} \partial_{x} J_{\varepsilon}\left(\partial_{x} u\right)^{2}, \Lambda^{s} J_{\varepsilon} u\right\rangle_{L^{2}}
\end{aligned}
$$


where $\Lambda^{s}=\left(1-\partial_{x}^{2}\right)^{s / 2}$. The first term on the right hand side can be bounded as in [T2], see p. $115-116$

$$
\left\langle\Lambda^{s} J_{\varepsilon}\left(u \partial_{x} u\right), \Lambda^{s} J_{\varepsilon} u\right\rangle_{L^{2}} \lesssim\|u\|_{C^{1}}\|u\|_{H^{s}}^{2} .
$$

For the middle term we have

$$
\left\langle\Lambda^{s} A^{-1} \partial_{x} J_{\varepsilon}(\mu(u) u), \Lambda^{s} J_{\varepsilon} u\right\rangle_{L^{2}} \lesssim|\mu(u)|\left\|\Lambda^{s} A^{-1} \partial_{x} J_{\varepsilon} u\right\|_{L^{2}}\left\|\Lambda^{s} J_{\varepsilon} u\right\|_{L^{2}} \lesssim\|u\|_{L^{1}}\|u\|_{H^{s}}^{2} .
$$

To estimate the last term we again use Cauchy-Schwarz

$$
\left\langle\Lambda^{s} A^{-1} \partial_{x} J_{\varepsilon}\left(\partial_{x} u\right)^{2}, \Lambda^{s} J_{\varepsilon} u\right\rangle_{L^{2}} \lesssim\left\|A^{-1} \partial_{x}\left(\partial_{x} u\right)^{2}\right\|_{H^{s}}\|u\|_{H^{s}}
$$

and with the help of the identities (5.13) and (5.14) we obtain

$$
\begin{aligned}
\left\|A^{-1} \partial_{x}\left(\partial_{x} u\right)^{2}\right\|_{H^{s}} & \simeq\left\|A^{-1} \partial_{x}\left(\partial_{x} u\right)^{2}\right\|_{L^{2}}+\left\|\partial_{x} A^{-1} \partial_{x}\left(\partial_{x} u\right)^{2}\right\|_{H^{s-1}} \\
& \lesssim\left\|\partial_{x} u\right\|_{L^{2}}^{2}+\left\|-\left(\partial_{x} u\right)^{2}+\int\left(\partial_{x} u\right)^{2} d x\right\|_{H^{s-1}} \\
& \lesssim\left\|\partial_{x} u\right\|_{L^{2}}^{2}+\left\|\partial_{x} u\right\|_{\infty}\|u\|_{H^{s}}
\end{aligned}
$$

where in the last step we used a standard Moser inequality for products of $H^{s-1}$ functions.

Collecting these estimates and passing to the limit with $\varepsilon \rightarrow 0$ we arrive at the differential inequality

$$
\frac{d}{d t}\|u\|_{H^{s}}^{2} \lesssim\|u\|_{C^{1}}\|u\|_{H^{s}}^{2}
$$

which combined with Gronwall's inequality implies that the $H^{s}$ norm of the solution $u$ cannot blow up unless $\|u(t, \cdot)\|_{C^{1}}$ does.

5.2. Global solutions with non-negative angular momentum density. Our next result shows the effect of the term $2 \mu(u) u_{x}$ on the lifespan of solutions to the Cauchy problem.

Theorem 5.5. Let $s>5 / 2$. Assume that the function $u_{o} \in H^{s}\left(S^{1}\right)$ has non-zero mean and satisfies the condition

$$
A u_{o}(x):=\left(\mu-\partial_{x}^{2}\right) u_{o}(x) \geq 0, \quad x \in S^{1} .
$$

Then the Cauchy problem (5.1)-(5.2) has a unique global solution $u$ in the space $C\left(\mathbb{R}, H^{s}\right) \cap C^{1}\left(\mathbb{R}, H^{s-1}\right)$.

Proof In Theorem 5.1 for any such $u_{o}$ we constructed a local solution $u$ with desired regularity and defined up to some time $T>0$. Furthermore, we showed that in order to extend it beyond $T$ it suffices to find a constant $K>0$ such that $\|u(t, \cdot)\|_{C^{1}} \leq K$ for all $|t|<T$.

Set $m(t, x)=A u(t, x)$ and observe that from the identity (5.13) we have

$$
\partial_{x} u(t, x)=\left(x-\frac{1}{2}\right) \int m(t, x) d x+\iint_{0}^{x} m(t, y) d y d x-\int_{0}^{x} m(t, y) d y
$$

for any $x \in[0,1]$, which consequently yields a bound

$$
\left\|\partial_{x} u(t)\right\|_{\infty} \lesssim\|m(t)\|_{L^{1}} .
$$

On the other hand, it is not difficult to check that at each point of the circle the solution $u$ satisfies a local conservation law

$$
A u(t, \eta(t, x))\left(\partial_{x} \eta(t, x)\right)^{2}=A u_{o}(x),
$$


where $\eta$ is the geodesic in $\mathcal{D}^{s}\left(S^{1}\right)$ corresponding to $u$. In particular, if (5.17) holds then $m(t, x) \geq 0$ as long as $u(t, x)$ exists, which in turn yields

$$
\|m(t)\|_{L^{1}}=\int u(t, x) d x=\int u_{o}(x) d x
$$

and the theorem follows.

An analogous result holds if the assumption (5.17) is replaced with $A u_{o}(x) \leq 0$. Notice that for the periodic Camassa-Holm equation the condition that $u-u_{x x}$ does not properly change sign is necessary and sufficient for global existence, see [McK].

5.3. Breakdown of classical solutions. It is not difficult to find solutions which develop singularities in finite time. In fact, the conservation of the mean

$$
\int u(t, x) d x=\int u_{o}(x) d x
$$

implies that sufficiently smooth solutions of $\mu \mathrm{HS}$ corresponding to zero-mean initial data must satisfy the periodic HS equation

$$
u_{t x x}+2 u_{x} u_{x x}+u u_{x x x}=0,
$$

whose solutions inevitably break down. We recall here a simple proof of this fact 7 . Consider any smooth (non-constant) periodic solution $u$ of the HS equation with smooth initial data $u_{o}$ (see e.g. [Ti]). Integrating ( $(\underline{\mathrm{HS}})$ with respect to the space variable we obtain

where

$$
u_{t x}+\frac{1}{2} u_{x}^{2}+u u_{x x}=a
$$

$$
a=-\frac{1}{2} \int u_{x}^{2} d x
$$

is independent of time as is easily checked using periodicity and integration by parts. (Alternatively, one notices that $a$ is a multiple of the $\dot{H}^{1}$-energy of the solution, which is conserved for HS.) Since $u_{o}^{\prime}$ is continuous we can pick a point $x_{o}$ where $u_{o}^{\prime}\left(x_{o}\right)<0$. Then, along the characteristic $t \rightarrow x(t)$ defined by $x(0)=x_{o}$ we get an ordinary differential equation of the form

$$
\frac{d w}{d t}+\frac{1}{2} w^{2}=a, \quad \text { where } \quad w(t)=u_{x}(t, x(t))
$$

whose explicit solution

$$
w(t)=\frac{u_{o}^{\prime}\left(x_{o}\right) \sqrt{-2 a}-2 a \tan (-t \sqrt{-2 a} / 2)}{\sqrt{-2 a}+u_{o}^{\prime}\left(x_{o}\right) \tan (t \sqrt{-2 a} / 2)}
$$

becomes unbounded as $t \nearrow T_{\text {crit }}=\frac{2}{\sqrt{-2 a}} \arctan \left(\sqrt{-2 a} /\left|u_{o}^{\prime}\left(x_{o}\right)\right|\right)$.

Note, however, that blow-up of $\mu \mathrm{HS}$ solutions can occur even if the mean of the initial data is not zero. More precisely, if $u$ is a smooth solution of (5.1)-(5.2) then proceeding as above we arrive at the equation

$$
u_{t x}-2 \mu(u) u+\frac{1}{2} u_{x}^{2}+u u_{x x}=r_{0},
$$

where the constant of integration

$$
r_{0}=-2 \mu(u)^{2}-\frac{1}{2} \int u_{x}^{2} d x
$$

\footnotetext{
${ }^{7}$ This argument was worked out by K. Gleason in her Senior Thesis [Gl]; see also [Y].
} 
is again independent of time. Using Cauchy-Schwarz and conservation of the energy $2 H_{1}(u)=\mu(u)^{2}+\int u_{x}^{2} d x$ and the mean (5.19), we then estimate

$$
\begin{aligned}
u_{t x}+\frac{1}{2} u_{x}^{2}+u u_{x x} & =r_{0}+2 \mu(u) u \\
& =2 \mu\left(u_{o}\right)\left(u(t, x)-\int u(t, x) d x\right)-\frac{1}{2} \int\left(u_{o}^{\prime}\right)^{2} d x \\
& \leq\left(2\left|\mu\left(u_{o}\right)\right|-\frac{1}{2}\left\|u_{o}^{\prime}\right\|_{L^{2}}\right)\left\|u_{o}^{\prime}\right\|_{L^{2}}
\end{aligned}
$$

since the function $u-\mu(u)$ must vanish somewhere on the circle. The conservation law $H_{1}$ ensures that solutions remain bounded as long as they are defined. Therefore the characteristics are defined as long as the solution exists. If we arrange for the right-hand side above to be non-positive then along any characteristic the function $w(t)=u_{x}(t, x(t))$ will satisfy

$$
\frac{d w}{d t} \leq-\frac{1}{2} w^{2}
$$

and so picking $x_{o}$ as before with $u_{o}^{\prime}\left(x_{o}\right)<0$ we get

$$
w(t) \leq w(0)=u_{o}^{\prime}\left(x_{o}\right)<0
$$

as long as it exists. Using this and solving (5.20) we therefore obtain for any $t \geq 0$ the estimate

$$
w(t) \leq \frac{2 u_{o}^{\prime}\left(x_{o}\right)}{2+t u_{o}^{\prime}\left(x_{o}\right)},
$$

from which conclude that $w(t)$ becomes infinite at $T_{\text {crit }}=2 /\left|u_{o}^{\prime}\left(x_{o}\right)\right|$. We summarize these results in

Proposition 5.6. For any smooth initial data satisfying $4\left|\mu\left(u_{o}\right)\right| \leq\left\|u_{o}^{\prime}\right\|_{L^{2}}$ there exists $T_{\text {crit }}>0$ such that the corresponding solution $u(t, x)$ of the $\mu H S$ equation remains bounded for $t<T_{\text {crit }}$ and satisfies $\left\|u_{x}(t)\right\|_{\infty} \nearrow \infty$ whenever $t \nearrow T_{\text {crit }}$.

Thus, the $\mu \mathrm{HS}$ equation is an example of a nonlinear PDE admitting solutions with finite as well as infinite lifespan 8 . As a simple illustration, the solution corresponding to the initial data $u_{o}(x)=a+\cos 2 \pi x$ blows up whenever $|a| \leq \pi \sqrt{2} / 4$ while persisting indefinitely if $|a| \geq 4 \pi^{2}$.

It seems likely that Theorem 5.5 and Proposition 5.6 can be significantly strengthened to give the full picture of well-posedness including a complete characterization of the blow-up mechanism (e.g. along the lines of [McK] or by studying the evolution of the extrema of $u_{x}$ as in [CE2]).

\section{Traveling-Wave Solutions}

In order to find traveling-wave solutions of the $\mu \mathrm{HS}$ equation we first regard $\mu$ as a parameter independent of $u$. We look for solutions $u(t, x)=\varphi(x-c t)$, traveling with speed $c \in \mathbb{R}$, of the equation

$$
-u_{t x x}=-2 \mu u_{x}+2 u_{x} u_{x x}+u u_{x x x},
$$

where $\mu \in \mathbb{R}$ is a parameter. A first analysis will give us a list of all traveling waves of this equation. Subsequently, by keeping only the solutions $\varphi$ for which the parameter $\mu$ equals the mean $\mu(\varphi)=\int \varphi d x$ of $\varphi$, the list will be narrowed down to traveling waves of the true $\mu$ HS equation. Since $\mu(\varphi)$ is a conserved quantity, this procedure yields all traveling waves of $\mu \mathrm{HS}$.

\footnotetext{
${ }^{8}$ Results of this type for the $\mathrm{CH}$ equation can be found e.g. in $\mathrm{CE}$.
} 
Substituting $u(t, x)=\varphi(x-c t)$ into equation (6.1) and integrating, we get

$$
c \varphi_{x x}=-2 \mu \varphi+\varphi \varphi_{x x}+\frac{1}{2} \varphi_{x}^{2}+\frac{a}{2},
$$

for some constant $a \in \mathbb{R}$. Rewriting this as

$$
\varphi_{x}^{2}=-4 \mu \varphi+\left((\varphi-c)^{2}\right)_{x x}+a .
$$

we have an equation that makes sense for $\varphi \in H_{l o c}^{1}(\mathbb{R})$ and it is natural to make the following definition: A function $\varphi \in H_{l o c}^{1}(\mathbb{R})$ is a traveling wave of equation ( $\mu H S$ ) if it satisfies (6.2) in distributional sense for some $a \in \mathbb{R}$. Applying the following lemma with $v=\varphi-c$ we immediately deduce that any traveling-wave solution $\varphi$ is smooth except possibly on the boundary of the set $\{x \in \mathbb{R} \mid \varphi(x)=c\}$. A general traveling-wave solution $\varphi(x)$ is therefore smooth on the union of the (possibly countably infinite) number of open intervals that make up the complement of this boundary.

Lemma 6.1. ([L]) Let $p(t)$ be a polynomial with real coefficients. Assume that $v \in$ $H_{\text {loc }}^{1}(\mathbb{R})$ satisfies

$$
\left(v^{2}\right)_{x x}=v_{x}^{2}+p(v) \quad \text { in } \quad \mathcal{D}^{\prime}(\mathbb{R}) .
$$

Then $v^{s} \in C^{j}(\mathbb{R})$ for $k \geq 2^{j}$. In particular, $v$ is smooth away from the boundary of the set $\{x \in \mathbb{R} \mid v(x)=0\}$.

Within each interval where $\varphi$ is smooth we may integrate (6.2) once more to obtain the ODE

$$
\varphi_{x}^{2}=\frac{-2 \mu \varphi^{2}+a \varphi+b}{c-\varphi}
$$

where $b \in \mathbb{R}$ is another integration constant which may take on different values for different intervals. If the roots of the polynomial $-2 \mu \varphi^{2}+a \varphi+b$ have nonzero imaginary part, there are no bounded solutions of (6.4). Thus, we may introduce real parameter $m$ and $M$ such that $m<M$ and

$$
-2 \mu \varphi^{2}+a \varphi+b=2 \mu(M-\varphi)(\varphi-m) .
$$

The next theorem follows from an analysis of (6.4) along the lines of [L]. Note that (6.2) is left unchanged under the substitutions

$$
\mu \mapsto-\mu, \quad \varphi \mapsto-\varphi, \quad c \mapsto-c,
$$

so it is enough to consider the case $\mu>0$.

Theorem 6.2. Let $\mu>0$. For a given $c \in \mathbb{R}$, the bounded traveling-wave solutions of speed $c$ can be classified by the values of the parameters $m, M \in \mathbb{R}$ as follows.

(a) (Smooth periodic) If $m<M<c$, there is a smooth periodic traveling wave $\varphi(x-c t)$ of (6.1) with $\min _{x \in \mathbb{R}} \varphi(x)=m$ and $\max _{x \in \mathbb{R}} \varphi(x)=M$.

(b) (Cusped periodic) If $m<c<M$, there is a cusped periodic traveling wave $\varphi(x-c t)$ of (6.1) with $\min _{x \in \mathbb{R}} \varphi(x)=m$ and $\max _{x \in \mathbb{R}} \varphi(x)=c$.

(c) (Anticusped periodic) If $c<m<M$, there is an anticusped periodic traveling wave $\varphi(x-c t)$ of (6.1) with $\min _{x \in \mathbb{R}} \varphi(x)=c$ and $\max _{x \in \mathbb{R}} \varphi(x)=m$.

(d) (Anticusped on the line) If $c<m=M$, there is an anticusped traveling wave $\varphi(x-c t)$ of (6.1) with $\min _{x \in \mathbb{R}} \varphi(x)=c$ and $\lim _{x \rightarrow \pm \infty} \varphi(x)=\max _{x \in \mathbb{R}} \varphi(x)=$ $m=M$. 
(e) (Composite waves) Two wave-segments in $(a)-(d)$ corresponding to parameters $\left(m_{1}, M_{1}\right)$ respectively $\left(m_{2}, M_{2}\right)$ are allowed to be glued together at their crests whenever $m_{1}+M_{1}=m_{2}+M_{2}$. Any composite wave formed by joining at most countably many wave-segments in this manner is a traveling wave of (6.1).

(f) (Plateaus) In the special case when $m_{1}+M_{1}=m_{2}+M_{2}=c$, the composite wave may also contain plateaus, i.e. intervals on which $\varphi \equiv c$.

6.1. Solitary waves. From Theorem 6.2 we infer the following list of solitary waves (i.e. traveling waves with decay to zero at infinity) of equation (6.1).

- If $\mu>0$, then, for each $c<0$, there is an anticusped solitary wave $\varphi(x-c t)$ with $\min _{x \in \mathbb{R}} \varphi(x)=c$ and $\lim _{x \rightarrow \pm \infty} \varphi(x)=0$.

- If $\mu<0$, then, for each $c>0$, there is a cusped solitary wave $\varphi(x-c t)$ with $\lim _{x \rightarrow \pm \infty} \varphi(x)=0$ and $\max _{x \in \mathbb{R}} \varphi(x)=c$.

To verify that these are indeed anticusped respectively cusped solitary waves, we may determine their profiles directly. For example, choosing the case $\mu<0$ so that $c>0$, we employ (6.4) with integration constants $a$ and $b$ set to zero to get

$$
\int \sqrt{\frac{c-\varphi}{-2 \mu \varphi^{2}}} d \varphi=x-x_{0}
$$

The integral can be computed to yield the formula

$$
\sqrt{\frac{2}{-\mu}}\left(\sqrt{c-\varphi}-\sqrt{c} \operatorname{arctanh}\left(\sqrt{\frac{c-\varphi}{c}}\right)\right)=x-x_{0} .
$$

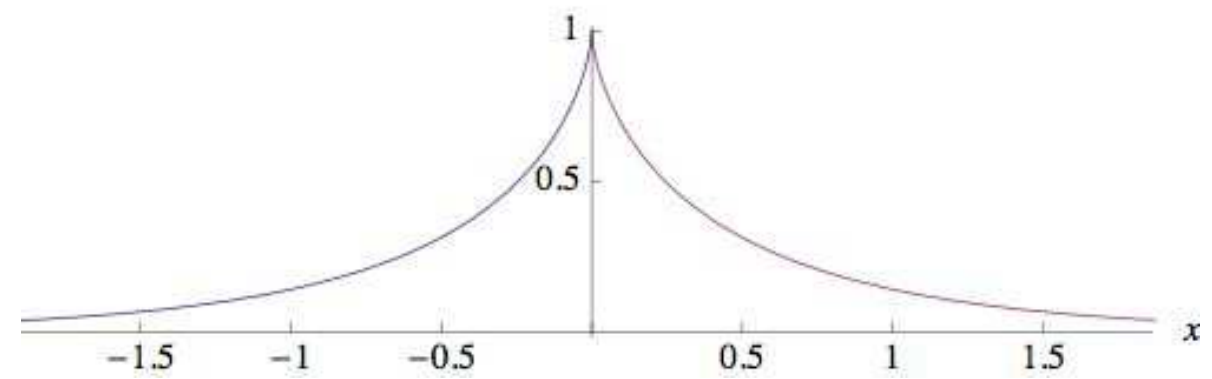

FigURE 1. Although this cusped solitary wave is a solution moving with speed $c=1$ of equation (6.1) when $\mu=-1$, it is not a solution of the $\mu \mathrm{HS}$ equation due to the negative sign of $\mu$.

Note that $x \rightarrow-\infty$ as $\varphi \searrow 0$ and that $x-x_{0} \rightarrow 0$ as $\varphi \nearrow c$. Near the crest point we get

$$
\frac{\sqrt{2}}{3 c \sqrt{-\mu}}(c-\varphi)^{3 / 2}+O\left((c-\varphi)^{5 / 2}\right)=\left|x-x_{0}\right|,
$$

and so, near $x=x_{0}$,

$$
c-\varphi(x)=\left(\frac{3 c \sqrt{-\mu}}{\sqrt{2}}\right)^{2 / 3}\left|x-x_{0}\right|^{2 / 3}+O\left(\left|x-x_{0}\right|^{4 / 3}\right),
$$

showing that there is indeed a cusp at $x=x_{0}$.

Do any of these solitary waves satisfy the condition that $\mu$ equals $\int_{\mathbb{R}} \varphi d x$, so that they are also solutions of the $\mu \mathrm{HS}$ equation? The answer is no. Indeed, if $\mu>0$, 
then since $\max _{x \in \mathbb{R}} \varphi(x)=0$, the solitary wave has $\varphi \leq 0$ everywhere, and so $\mu(\varphi)=$ $\int_{\mathbb{R}} \varphi d x \leq 0$ never equals $\mu$. The case $\mu<0$ is analogous.

Theorem 6.3. The $\mu H S$ equation does not have solitary wave solutions on the line.

There are also composite solitary wave solutions obtained by inserting cusped or anticusped periodic wave segments at the crests of these solitary waves according to (e) in Theorem 6.2. One might hope that a clever construction of a composite wave would yields a different outcome. However, since $m+M=0$ for the solitary wave and $m<M$ by definition, one can see that any cusped wave segments joined to the solitary wave must have the same sign, and thus the sign of the integral $\int_{\mathbb{R}} \varphi d x$ remains unchanged.

This analysis shows that the $\mu \mathrm{HS}$ equations admits no solitary waves, and hence no solitons, on the line. In the periodic setting for $\mu \mathrm{HS}$, however, we will see that traveling waves which are natural candidates for solitons do exist.

6.2. Traveling waves on the circle. In view of the symmetry (6.6) we restrict ourselves to the case $\mu>0$ in this section.

Consider equation (6.4)

$$
\varphi_{x}^{2}=\frac{2 \mu(M-\varphi)(\varphi-m)}{c-\varphi},
$$

written in terms of $m$ and $M$ according to (6.5). By (a), (b), and (c) of Theorem 6.2 there is a unique periodic traveling wave $\varphi_{m, M, \mu}$ of equation (6.1) associated to each point $(m, M, \mu)$ such that (a) $m<M<c$, (b) $m<c<M$, or (c) $c<m<M$. By enforcing the constraint

$$
\operatorname{mean}\left(\varphi_{m, M, \mu}\right)=\mu
$$

we obtain the subset of these that are also solutions of the $\mu \mathrm{HS}$ equation. From the way $\mu$ appears in (6.7) we infer that (see (6.12) below)

$$
\operatorname{mean}\left(\varphi_{m, M, \mu}\right)=\frac{1}{\sqrt{\mu}} \operatorname{mean}\left(\varphi_{m, M, 1}\right) .
$$

The constraint (6.8) therefore translates to mean $\left(\varphi_{m, M, 1}\right)=\mu^{3 / 2}$ and is seen to admit a unique solution $\mu>0$ exactly when mean $\left(\varphi_{m, M, 1}\right)>0$. Whereas this condition fails to hold in the case of solitary waves as noted above, it is satisfied for large classes of periodic waves. Rather than finding the most general choices of $m, M$, and $c$ for which it is fulfilled, we simply note that if the minimum of $\varphi_{m, M, 1}$ is positive then its mean is certainly also positive. Applying Theorem 6.2 with $\mu=1$ we can therefore deduce existence of the following classes of traveling waves parametrized by $m, M, c \in \mathbb{R}$.

(a) (Smooth periodic) Whenever $0<m<M<c$, there is a smooth periodic traveling wave $\varphi(x-c t)$ of $\mu \mathrm{HS}$ with $\min _{x \in \mathbb{R}} \varphi(x)=m$ and $\max _{x \in \mathbb{R}} \varphi(x)=M$.

(b) (Cusped periodic) Whenever $0<m<c<M$, there is a cusped periodic traveling wave $\varphi(x-c t)$ of $\mu \mathrm{HS}$ with $\min _{x \in \mathbb{R}} \varphi(x)=m$ and $\max _{x \in \mathbb{R}} \varphi(x)=c$.

(c) (Anticusped periodic) Whenever $0<c<m<M$, there is an anticusped periodic traveling wave $\varphi(x-c t)$ of $\mu \mathrm{HS}$ with $\min _{x \in \mathbb{R}} \varphi(x)=c$ and $\max _{x \in \mathbb{R}} \varphi(x)=$ $m$.

In the rest of this section we will derive explicit expressions (involving elliptic integrals) for the period and the mean of these waves. As a consequence of the analysis below, we will see that there are plenty of traveling waves of period one, i.e. on the circle $S^{1}=\mathbb{R} / \mathbb{Z}$. 
Theorem 6.4. For any $c \neq 0$ the $\mu H S$ equation has a one-parameter family of smooth periodic traveling waves of period one and a one-parameter family of cusped periodic traveling waves of period one moving with speed $c$.

6.2.1. Smooth periodic waves. From (6.7) it follows that

$$
x-x_{0}=\frac{1}{\sqrt{2 \mu}} \int_{\varphi_{0}}^{\varphi} \sqrt{\frac{c-\varphi}{(M-\varphi)(\varphi-m)}} d \varphi .
$$

Substituting $\varphi=m+(M-m) \sin ^{2} \theta$, we find

$$
x-x_{0}=\sqrt{\frac{2(c-m)}{\mu}} \int_{\theta_{0}}^{\theta} \sqrt{1-\frac{M-m}{c-m} \sin ^{2} \theta} d \theta=\left.\sqrt{\frac{2(c-m)}{\mu}} E\left(\theta \mid \frac{M-m}{c-m}\right)\right|_{\theta_{0}} ^{\theta},
$$

which expresses $x-x_{0}$ in terms of the incomplete elliptic integral of the second kind

$$
E(\phi \mid r):=\int_{0}^{\phi} \sqrt{1-r \sin ^{2} \theta} d \theta, \quad-\frac{\pi}{2}<\phi<\frac{\pi}{2} .
$$

Now for smooth periodic waves the period is given by twice the integral from the minimum $\varphi_{0}=m$ to the maximum $\varphi_{1}=M$, or, in terms of $\theta$, from $\theta_{0}=0$ to $\theta_{1}=\frac{\pi}{2}$. We deduce that

$$
\operatorname{period}\left(\varphi_{m, M, \mu}\right)=2 \sqrt{\frac{2(c-m)}{\mu}} E\left(\frac{M-m}{c-m}\right)
$$

where $E(r):=E(\pi / 2 \mid r)$ is the complete elliptic integral of the second kind.

Similarly, we compute

$$
\begin{gathered}
\int_{x_{0}}^{x} \varphi d x=\frac{1}{\sqrt{2 \mu}} \int_{\varphi_{0}}^{\varphi} \varphi \sqrt{\frac{c-\varphi}{(M-\varphi)(\varphi-m)}} d \varphi \\
=\sqrt{\frac{2(c-m)}{\mu} \int_{\theta_{0}}^{\theta}\left(m+(M-m) \sin ^{2} \theta\right) \sqrt{1-\frac{M-m}{c-m} \sin ^{2} \theta} d \theta .} .
\end{gathered}
$$

Then integration over a full period yields

$$
\operatorname{mean}\left(\varphi_{m, M, \mu}\right)=\frac{2}{3} \sqrt{\frac{2(c-m)}{\mu}}\left((2(m+M)-c) E\left(\frac{M-m}{c-m}\right)+(c-M) K\left(\frac{M-m}{c-m}\right)\right),
$$

where

$$
K(r):=\int_{0}^{\pi / 2}\left(1-r \sin ^{2} \theta\right)^{-1 / 2} d \theta
$$

is the complete elliptic integral of the first kind. Observe that the mean indeed scales with $\mu$ in accordance with (6.9).

Solving the constraint (6.8) for $\mu$ we see that the mean of the traveling wave $\varphi$ of the $\mu \mathrm{HS}$ equation associated to the point $(m, M)$ is

$$
\mu(\varphi)=\left(\frac{2}{3} \sqrt{2(c-m)}\left((2(m+M)-c) E\left(\frac{M-m}{c-m}\right)+(c-M) K\left(\frac{M-m}{c-m}\right)\right)\right)^{2 / 3}
$$

and substituting this value of $\mu$ into (6.11), we find that the corresponding period is $(6.13)$

$$
\operatorname{period}(\varphi)=\frac{2 \cdot 3^{1 / 3} \sqrt{c-m} E\left(\frac{M-m}{c-m}\right)}{\left(\sqrt{c-m}\left((2(m+M)-c) E\left(\frac{M-m}{c-m}\right)+(c-M) K\left(\frac{M-m}{c-m}\right)\right)\right)^{1 / 3}} .
$$




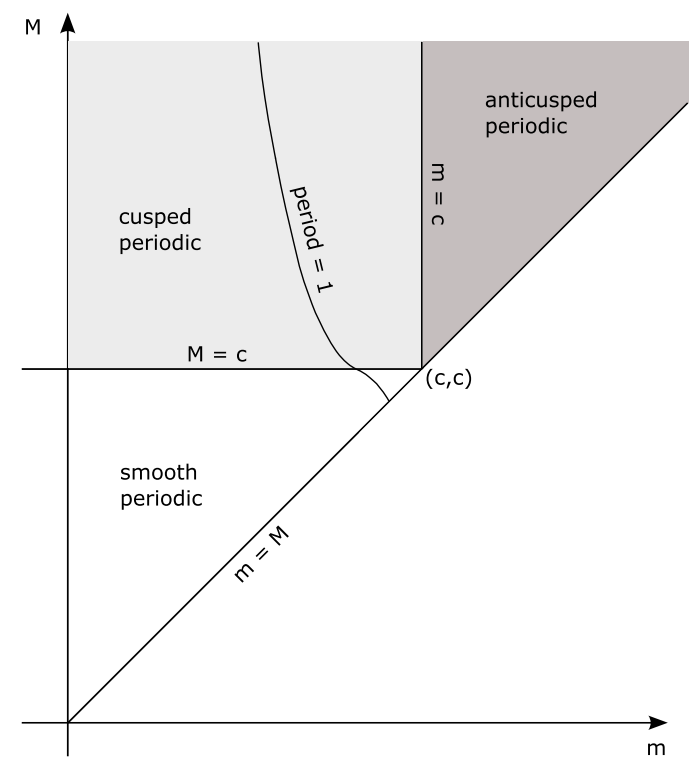

Figure 2. For each $c \neq 0$ there are one-parameter families of both smooth and cusped period-one traveling waves of the $\mu \mathrm{HS}$ equation.

6.2.2. Cusped periodic waves. For cusped periodic waves $(m<c<M)$ the period is given by twice the integral (6.10) from $\varphi_{0}=m$ to $\varphi_{1}=c$, i.e. from $\theta_{0}=0$ to $\theta_{1}=\arcsin \left(\sqrt{\frac{c-m}{M-m}}\right)$. We get

$$
\operatorname{period}\left(\varphi_{m, M, \mu}\right)=2 \sqrt{\frac{2(c-m)}{\mu}} E\left(\arcsin \left(\sqrt{\frac{c-m}{M-m}}\right) \mid \frac{M-m}{c-m}\right) .
$$

Similarly, formula (6.12) for mean $\left(\varphi_{m, M, \mu}\right)$ and formula (6.13) for the period also hold in the cusped case with the replacements

$$
E\left(\frac{M-m}{c-m}\right) \rightarrow E\left(\arcsin \left(\sqrt{\frac{c-m}{M-m}}\right) \mid \frac{M-m}{c-m}\right)
$$

and

$$
K\left(\frac{M-m}{c-m}\right) \rightarrow F\left(\arcsin \left(\sqrt{\frac{c-m}{M-m}}\right) \mid \frac{M-m}{c-m}\right)
$$

where

$$
F(\phi \mid r):=\int_{0}^{\phi}\left(1-r \sin ^{2} \theta\right)^{-1 / 2} d \theta
$$

is the incomplete analog of $K$. An analysis of the expression for the period in (6.13), together with its cusped analog, shows that the level set of period-one solutions looks qualitatively as in Figure 2. In particular, we see that for each $c>0$ there are oneparameter families of both smooth and cusped period-one traveling-wave solutions of the $\mu \mathrm{HS}$ equation. This establishes Theorem 6.4.

\section{Geometry of the Group of CIRCLe Diffeomorphisms}

The objective of this section is to compute the curvature of $\mathcal{D}^{s}\left(S^{1}\right)$ endowed with the $\mu \mathrm{HS}$ metric (2.2). We establish a local formula for a connection compatible with the metric and use it to obtain an exact expression for the curvature tensor. In 
particular, we prove that sectional curvatures in both vertical and horizontal twodimensional directions on $\mathcal{D}^{s}\left(S^{1}\right)$ are positive.

7.1. Christoffel symbols and the covariant derivative. Define a symmetric bilinear map $\Gamma_{i d}(\cdot, \cdot)$ on $T_{i d} \mathcal{D}^{s}\left(S^{1}\right) \simeq H^{s}\left(S^{1}\right)$ by

$$
\Gamma_{i d}(u, v)=-A^{-1} \partial_{x}\left(\mu(u) v+\mu(v) u+\frac{1}{2} u_{x} v_{x}\right), \quad u, v \in H^{s}\left(S^{1}\right),
$$

and extend it to a bilinear map $\Gamma_{\eta}(\cdot, \cdot): T_{\eta} \mathcal{D}^{s}\left(S^{1}\right) \times T_{\eta} \mathcal{D}^{s}\left(S^{1}\right) \rightarrow T_{\eta} \mathcal{D}^{s}\left(S^{1}\right)$ for any $\eta \in \mathcal{D}^{s}\left(S^{1}\right)$ using right invariance.

Next, define the operator $\nabla$ locally in a chart at $\eta$ by the formula

$$
\left(\nabla_{X} Y\right)(\eta)=D Y(\eta) \cdot X(\eta)-\Gamma_{\eta}(Y(\eta), X(\eta))
$$

for any vector fields $X$ and $Y$ on $\mathcal{D}^{s}\left(S^{1}\right)$. It turns out that $\nabla$ is the smooth LeviCivita connection of the right-invariant $\mu \mathrm{HS}$ metric whose Christoffel symbols are given by the map $\Gamma$. The proof of the following proposition is straightforward (see for example [EM]).

Proposition 7.1. The bilinear map $\nabla$ defined by (7.2) is the unique torsion-free covariant derivative on $\mathcal{D}^{s}\left(S^{1}\right)$ compatible with the right-invariant metric (2.2).

7.2. Sectional curvature. In order to compute the sectional curvature of $\mathcal{D}^{s}\left(S^{1}\right)$ equipped with the $\mu \mathrm{HS}$ metric $\langle\cdot, \cdot\rangle$ defined in (2.2) recall first that its curvature tensor $R$ can be determined from the formula

$$
R(X, Y) Z=\nabla_{X} \nabla_{Y} Z-\nabla_{Y} \nabla_{X} Z-\nabla_{[X, Y]} Z
$$

for any vector fields $X, Y$ and $Z$ on $\mathcal{D}^{s}\left(S^{1}\right)$. By right invariance it will be sufficient to compute $R$ at the identity. We will write $\Gamma(u, v)$ to denote the Christoffel symbol $\Gamma_{i d}(u, v)$.

Theorem 7.2. For any $u, v \in T_{i d} \mathcal{D}^{s}\left(S^{1}\right) \simeq H^{s}\left(S^{1}\right)$, it holds that

$$
\begin{aligned}
\langle R(u, v) v, u\rangle= & \langle\Gamma(u, v), \Gamma(u, v)\rangle-\langle\Gamma(u, u), \Gamma(v, v)\rangle-3 \mu\left(u_{x} v\right)^{2} \\
= & \mu(u)^{2}\left(\mu\left(v^{2}\right)+\mu\left(v_{x}^{2}\right)\right)+\mu(v)^{2}\left(\mu\left(u^{2}\right)+\mu\left(u_{x}^{2}\right)\right) \\
& +\mu(u) \mu\left(\left(v u_{x}-u v_{x}\right) v_{x}\right)+\mu(v) \mu\left(\left(u v_{x}-v u_{x}\right) u_{x}\right) \\
& -2 \mu(u) \mu(v)\left(\mu(u v)+\mu\left(u_{x} v_{x}\right)\right) \\
& -\frac{1}{4}\left(\int u_{x} v_{x} d x\right)^{2}+\frac{1}{4} \int u_{x}^{2} d x \int v_{x}^{2} d x-3 \mu\left(u_{x} v\right)^{2} .
\end{aligned}
$$

Proof For any $u, v$ and $w \in T_{i d}^{s} \mathcal{D}\left(S^{1}\right) \simeq H^{s}\left(S^{1}\right)$ the local formula corresponding to the expression in (7.3) reads

$$
\begin{aligned}
R(u, v) w= & D_{1} \Gamma_{i d}(w, u) v-D_{1} \Gamma_{i d}(w, v) u \\
& +\Gamma_{\eta}\left(\Gamma_{i d}(w, v), u\right)-\Gamma_{i d}\left(\Gamma_{\eta}(w, u), v\right)
\end{aligned}
$$

where $D_{1}$ denotes differentiation of $\Gamma_{\eta}$ with respect to $\eta$ : $D_{1} \Gamma_{i d}(w, u) v=\left.\frac{d}{d \epsilon}\right|_{\epsilon=0} \Gamma_{i d+\epsilon v}(w, u)$. We compute

$$
D_{1} \Gamma_{i d}(w, u) v=-\Gamma_{i d}\left(w_{x} v, u\right)-\Gamma_{i d}\left(w, u_{x} v\right)+\Gamma_{i d}(w, u)_{x} v
$$

and so

$$
\begin{aligned}
\langle R(u, v) v, u\rangle= & \left\langle-\Gamma\left(v_{x} v, u\right)-\Gamma\left(v, u_{x} v\right)+\Gamma\left(v_{x} u, v\right)+\Gamma\left(v, v_{x} u\right), u\right\rangle \\
& +\left\langle\Gamma(v, u)_{x} v-\Gamma(v, v)_{x} u+\Gamma(\Gamma(v, v), u)-\Gamma(\Gamma(v, u), v), u\right\rangle .
\end{aligned}
$$


Observe that

$$
\Gamma(u, v)=\frac{1}{2}\left((u v)_{x}-a d_{v}^{*}(u)-a d_{u}^{*}(v)\right),
$$

where $a d_{v}^{*}(u)=A^{-1}\left(2 v_{x} A u+v A u_{x}\right)$, see (2.4). Furthermore, note that the last four terms in the expression for $\langle R(u, v) v, u\rangle$ can be rewritten as

$$
\begin{gathered}
\left\langle\Gamma(v, u)_{x}-\Gamma(v, v)_{x} u+\Gamma(\Gamma(v, v), u)-\Gamma(\Gamma(v, u), v), u\right\rangle \\
=\langle\Gamma(u, v), \Gamma(u, v)\rangle-\langle\Gamma(u, u), \Gamma(v, v)\rangle \\
-\left\langle u_{x} v, \Gamma(v, u)\right\rangle+\left\langle u u_{x}, \Gamma(v, v)\right\rangle .
\end{gathered}
$$

Therefore

$$
\begin{aligned}
\langle R(u, v) v, u\rangle= & \left\langle-\Gamma\left(v_{x} v, u\right)-\Gamma\left(v, u_{x} v\right)+\Gamma\left(v_{x} u, v\right)+\Gamma\left(v, v_{x} u\right), u\right\rangle \\
& +\langle\Gamma(u, v), \Gamma(u, v)\rangle-\langle\Gamma(u, u), \Gamma(v, v)\rangle \\
& -\left\langle u_{x} v, \Gamma(v, u)\right\rangle+\left\langle u u_{x}, \Gamma(v, v)\right\rangle .
\end{aligned}
$$

Now, using the formula for the Christoffel symbols in (7.1) a straightforward computation gives

$$
\begin{aligned}
\left\langle-\Gamma\left(v_{x} v, u\right)-\Gamma\left(v, u_{x} v\right)+\Gamma\left(v_{x} u, v\right)+\Gamma\left(v, v_{x} u\right), u\right\rangle & -\left\langle u_{x} v, \Gamma(v, u)\right\rangle+\left\langle u u_{x}, \Gamma(v, v)\right\rangle \\
& =-3\left(\int u_{x} v d x\right)^{2} .
\end{aligned}
$$

This establishes the first curvature formula of Theorem 7.2 .

To obtain the second equality in (7.4) we simply substitute the expression for the Christoffel symbol $\Gamma$ into

$$
\langle\Gamma(u, v), \Gamma(u, v)\rangle-\langle\Gamma(u, u), \Gamma(v, v)\rangle-3\left(\int u_{x} v d x\right)^{2}
$$

and integrate by parts to get

$$
\begin{aligned}
& -\int\left(\mu(u) v+\mu(v) u+\frac{1}{2} u_{x} v_{x}\right) \partial_{x} A^{-1} \partial_{x}\left(\mu(u) v+\mu(v) u+\frac{1}{2} u_{x} v_{x}\right) d x \\
& +\int\left(2 \mu(u) u+\frac{1}{2} u_{x}^{2}\right) \partial_{x} A^{-1} \partial_{x}\left(2 \mu(v) v+\frac{1}{2} v_{x}^{2}\right) d x-3\left(\int u_{x} v d x\right)^{2} .
\end{aligned}
$$

Using the identity $\partial_{x} A^{-1} \partial_{x} f=A^{-1} \partial_{x}^{2}=-f+\mu(f)$ we simplify this expression and obtain the right-hand side of (7.4).

From Theorem 7.2 we can immediately deduce an expression for the sectional curvature $K(u, v)$ of the plane spanned by two vectors $u, v \in T_{i d} \mathcal{D}^{s}\left(S^{1}\right)$. For this purpose we may assume, after taking linear combinations, that $u$ and $v$ are orthonormal with respect to the $\mu \mathrm{HS}$ metric $\langle\cdot, \cdot\rangle$ and that $\mu(v)=0$, i.e.

$$
\mu(u)^{2}+\int_{S^{1}} u_{x}^{2} d x=1, \quad \mu(v)=0, \quad \int_{S^{1}} v_{x}^{2} d x=1, \quad \text { and } \quad \int_{S^{1}} u_{x} v_{x} d x=0 .
$$

With these assumptions, Theorem 7.2 yields

$$
K(u, v)=\mu(u)^{2}\left(\mu\left(v^{2}\right)+1\right)+\mu(u) \mu\left(\left(v u_{x}-u v_{x}\right) v_{x}\right)+\frac{1}{4} \int u_{x}^{2} d x-3 \mu\left(u_{x} v\right)^{2} .
$$

Proposition 7.3. For any orthonormal vectors $u, v \in T_{i d} \mathcal{D}^{s}\left(S^{1}\right)$ with $\mu(u)=\mu(v)=$ 0 the sectional curvature $K(u, v)$ of the plane spanned by $u$ and $v$ satisfies

$$
K(u, v)=\frac{1}{4}-3 \mu\left(u_{x} v\right)^{2} \geq \frac{1}{4}\left(1-\frac{3}{\pi^{2}}\right)>0 .
$$


Proof In this case (7.5) reduces to

$$
K(u, v)=\frac{1}{4}-3 \mu\left(u_{x} v\right)^{2} .
$$

By the Cauchy-Schwartz inequality it holds that

$$
\left(\int u_{x} v d x\right)^{2} \leq \int u_{x}^{2} d x \int v^{2} d x=\int v^{2} d x .
$$

Since $\mu(v)=0$ we also have

$$
\int v^{2} d x \leq \frac{1}{4 \pi^{2}} \int v_{x}^{2} d x=\frac{1}{4 \pi^{2}}
$$

so that (7.6) follows from (7.7).

Remark 7.4. The origin of the term $-3 \mu\left(u_{x} v\right)^{2}$ in (7.6) is as follows. For any $u, v \in T_{i d} \mathcal{D}^{s}\left(S^{1}\right)$ decomposed as $u=\tilde{u}+\mu(u)$ and $v=\tilde{v}+\mu(v)$ with $\mu(\tilde{u})=\mu(\tilde{v})=0$, Theorem 7.2 yields

$$
\langle R(u, v) v, u\rangle=\mu \text {-terms }+\left\langle R^{H S}(\tilde{u}, \tilde{v}) \tilde{v}, \tilde{u}\right\rangle-3 \mu\left(u_{x} v\right)^{2},
$$

where ' $\mu$-terms' stands for those terms containing either $\mu(u)$ or $\mu(v)$ (or both) as a factor, and

$$
\left\langle R^{H S}(\tilde{u}, \tilde{v}) \tilde{v}, \tilde{u}\right\rangle=-\frac{1}{4}\left(\int_{S^{1}} \tilde{u}_{x} \tilde{v}_{x} d x\right)^{2}+\frac{1}{4} \int_{S^{1}} \tilde{u}_{x}^{2} d x \int_{S^{1}} \tilde{v}_{x}^{2} d x
$$

is the curvature of the $\dot{H}^{1}$ metric (2.1) corresponding to the HS equation, see [L2]. Then the middle term in (7.8) corresponds to the curvature of the base $\mathcal{D}^{s}\left(S^{1}\right) / S^{1}$ contributing the constant term $\frac{1}{4}$ in (17.6), while the last term can be seen as the correction term in the general O'Neill formula $\mathrm{ON}$

$$
\left\langle R^{M}(X, Y) Y, X\right\rangle=\left\langle R^{B}(\tilde{X}, \tilde{Y}) \tilde{Y}, \tilde{X}\right\rangle-\frac{3}{4}\left\|[\tilde{X}, \tilde{Y}]^{V}\right\|^{2},
$$

for a Riemannian submersion $M \rightarrow B$, where $\tilde{X}, \tilde{Y}$ are vectors tangent to the base $B$, and $X, Y$ are their horizontal lifts to $M$. Indeed, for horizontal vectors $u$ and $v$ the $\mu$-terms in (77.8) vanish so that since $\mu\left(u_{x} v\right)^{2}=\mu([\tilde{u}, \tilde{v}])^{2} / 4$ the identification is clear.

To summarize, let us decompose the tangent space at the identity $T_{i d} \mathcal{D}^{s}\left(S^{1}\right)=$ $H \oplus V$ in such a way that $H$ consists of all mean zero functions on $S^{1}$ and $V \simeq \mathbb{R}$ are the constants (so that $u=\tilde{u}+\mu(u)$ with $\mu(\tilde{u})=0$ ). Proposition 7.3 shows that the sectional curvature for any plane contained in (i.e. parallel to) the subspace $H$ is strictly positive. Now turn to the planes perpendicular to $H$, i.e. planes containing constant functions, which constitute $V$.

Proposition 7.5. Let $v \in T_{i d} \mathcal{D}^{s}\left(S^{1}\right)$ be orthonormal to the constant function 1 . Then the sectional curvature of the plane spanned by 1 and $v$ is

$$
K(1, v)=\int v^{2} d x>0 .
$$

Proof Apply formula (7.5) with $u \equiv 1$ and $v$ satisfying $\mu(u)=1, u_{x} \equiv 0, \mu(v)=0$, and $\int_{S^{1}} v_{x}^{2} d x=1$. 
Remark 7.6. Applying Proposition [7.5 to the functions $\left\{v_{k}(x)=\frac{\sqrt{2} \sin k x}{k}\right\}_{k=1}^{\infty}$, we obtain a sequence of two-dimensional subspaces whose sectional curvatures $K\left(1, v_{k}\right)=$ $\frac{1}{k^{2}}$ approach zero as $k \rightarrow \infty$.

Remark 7.7. The group $\mathcal{D}^{s}\left(S^{1}\right)$ can be thought of as a solid torus, with a crosssection isomorphic to $\mathcal{D}^{s}\left(S^{1}\right) / S^{1}$. As we mentioned in the introduction, there is a natural Riemannian submersion of the metric $\langle\cdot, \cdot\rangle$ on $\mathcal{D}^{s}\left(S^{1}\right)$ to the $\dot{H}^{1}$-metric on $\mathcal{D}^{s}\left(S^{1}\right) / S^{1}$ defined in (2.1). The latter is isometric to a (contractible) piece $U \subset S^{\infty}$ of the unit sphere $S^{\infty}$ in $L^{2}\left(S^{1}\right)$, see [L2] 9 This set $U$ has a finite diameter, and any geodesic on it reaches the boundary of $U$ before acquiring a conjugate point.

This geometric picture sheds light on the (non)existence of global solutions proved in the section on well-posedness. Since the group $\mathcal{D}^{s}\left(S^{1}\right)$ with the metric $\langle\cdot, \cdot\rangle$ is a solid torus whose cross-section is a slight deformation of the open subset $U$ (of finite diameter), it is natural to expect that geodesics starting almost parallel to the crosssection (i.e. with small values $\mu(u)$ ) would run into the boundary of the solid torus (and only deviate slightly from the HS geodesics, which hit the boundary in short time). On the other hand, geodesics starting 'perpendicular' to the base would wind around the torus indefinitely and correspond to global solutions. This is exactly the picture which was made precise in Section 5 .

\section{8. $\mu$ HS AS A BI-HAMILTONIAN SYSTEM ON THE VIRASORO GROUP}

In Section 2 we showed that the $\mu \mathrm{HS}$ equation is the equation of the geodesic flow of the right-invariant metric (2.2) on the diffeomorphism group $\mathcal{D}^{s}\left(S^{1}\right)$, see Theorem 2.1. In other words, the $\mu \mathrm{HS}$ equation is the Euler equation on the Lie algebra $10 \mathfrak{v e c t}=T_{\text {id }} \mathcal{D}^{s}\left(S^{1}\right)$. By using the inner product (2.1) to identify vect with its dual algebra $\mathfrak{v e c t}^{*}$, the equation can be viewed as Hamiltonian with respect to the canonical Lie-Poisson structure and the Hamiltonian $H_{1}$.

In this section we shall carry out this construction on the Virasoro group, which is the universal central extension of $\mathcal{D}\left(S^{1}\right)$ or, equivalently, on the corresponding Lie algebra called the Virasoro algebra. As with the $\mathrm{KdV}$ and $\mathrm{CH}$ equations the Virasoro group and algebra provide a natural setting for a geometric interpretation of the biHamiltonian structure of the $\mu \mathrm{HS}$ equation discussed in Section 4, We will see that passing to the Virasoro algebra introduces an extra term $2 k u_{x}$. The corresponding Euler equation reads

$$
-u_{t x x}=-2 \mu(u) u_{x}+2 k u_{x}+2 u_{x} u_{x x}+u u_{x x x} \quad(k \in \mathbb{R}) .
$$

The situation is analogous to that of the $\mathrm{CH}$ equation which on the Virasoro algebra becomes (cf. $[\mathrm{KM}]$ )

$$
u_{t}-u_{t x x}+2 k u_{x}+3 u u_{x}=2 u_{x} u_{x x}+u u_{x x x} \quad(k \in \mathbb{R}) .
$$

In this case the parameter $k$ is related to the critical shallow water speed, see [J] 11

\footnotetext{
${ }^{9}$ One can show, however, that there is no isometric embedding of the group $\mathcal{D}^{s}\left(S^{1}\right)$ endowed with the $\mu \mathrm{HS}$ metric to $S^{\infty} \times S^{1}$, equipped with the direct product metric.

${ }^{10}$ Strictly speaking, $T_{\mathrm{id}} \mathcal{D}^{s}\left(S^{1}\right)$ may be considered a Lie algebra only when $s=\infty$. The reader can either make this assumption throughout the section or else take the Sobolev index $s$ sufficiently large for all constructions to be rigorously justified.

${ }^{11}$ One should also mention that in the case of the $\mathrm{CH}$ equation there is an important difference in regularity properties of the associated Riemannian exponential maps on the Virasoro and the diffeomorphism groups, see CKKT.
} 
Recall that the Virasoro algebra is the vector space $\mathfrak{v i r}=\mathfrak{v e c t} \oplus \mathbb{R}$ endowed with the following commutator between pairs

$$
[(v, b),(w, c)]=\left(v_{x} w-v w_{x}, \int v_{x} w_{x x} d x\right)
$$

where $v, w \in \mathfrak{v e c t}$ and $b, c \in \mathbb{R}$. Define an inner product on this algebra by

$$
\langle(v, b),(w, c)\rangle=\langle v, w\rangle+b c
$$

where $\langle\cdot, \cdot\rangle$ is the inner product in (2.1), and use it to identify the dual $\mathfrak{v i r}^{*}$ with $\mathfrak{v i r}$ by the pairing

$$
\langle(m, a),(v, b)\rangle^{*}=\int m v d x+a b
$$

where $(u, a) \mapsto(A u, a)=(m, a)$ is the corresponding inertia operator.

Let $h, f: \mathfrak{v i r}^{*} \rightarrow \mathbb{R}$ be arbitrary smooth functions. The dual space $\mathfrak{v i r}^{*}$ carries the canonical Lie-Poisson bracket

$$
\{h, f\}(m, a)=\left\langle(m, a),\left[\left(\frac{\delta h}{\delta m}, \frac{\delta h}{\delta a}\right),\left(\frac{\delta f}{\delta m}, \frac{\delta f}{\delta a}\right)\right]\right\rangle^{*} .
$$

On the other hand, given any point $\left(m_{0}, a_{0}\right)$ in $\mathfrak{v i r}^{*}$, we can also define

$$
\{h, f\}_{0}(m, a)=\left\langle\left(m_{0}, a_{0}\right),\left[\left(\frac{\delta h}{\delta m}, \frac{\delta h}{\delta a}\right),\left(\frac{\delta f}{\delta m}, \frac{\delta f}{\delta a}\right)\right]\right\rangle^{*}
$$

called a frozen (or constant) Poisson bracket. It is not difficult to check that both brackets form a Poisson pair in the sense that a linear combination of these brackets is again a Poisson bracket.

Our main result in this section is

Theorem 8.1. For any $k \in \mathbb{R}$ the $\mu H S$ equation (8.1) is Hamiltonian with respect to the two Poisson structures (8.3) and 8.4) on $\mathfrak{v i r}^{*}$ where the second bracket is frozen at the point $\left(m_{0}, a_{0}\right)=(0,1)$.

Proof From the relations

$$
\begin{aligned}
\left\langle a d_{(v, b)}^{*}(m, a),(w, c)\right\rangle^{*} & =\langle(m, a),[(v, b),(w, c)]\rangle^{*} \\
& =\left\langle(m, a),\left(v_{x} w-v w_{x}, \int v_{x} w_{x x} d x\right)\right\rangle^{*}
\end{aligned}
$$

holding for any elements $(m, a) \in \mathfrak{v i r}^{*}$ and $(v, b),(w, c) \in \mathfrak{v i r}$, we deduce an explicit form of the coadjoint action on $\mathfrak{v i r}^{*}$

$$
a d_{(v, b)}^{*}(m, a)=\left(m_{x} v+2 m v_{x}+a v_{x x x}, 0\right) .
$$

Since the Euler equation on $\mathfrak{v i r}^{*}$ is of the form $(m, a)_{t}=-a d_{(u, a)}^{*}(m, a)$ we obtain the system

$$
\left\{\begin{array}{l}
m_{t}=-m_{x} u-2 m u_{x}-a u_{x x x} \\
a_{t}=0 .
\end{array}\right.
$$

The second of the above equations immediately implies that $a$ must be constant and using $m=A u$ the first equation can be rewritten as

$$
-u_{t x x}=-2 \mu(u) u_{x}+2 u_{x} u_{x x}+u u_{x x x}-a u_{x x x} .
$$

Substituting $u \rightarrow u+a$ we recover the $\mu$ HS equation in the more familiar form (8.1) with the constant $k=-a$. Since the Euler equation on $\mathfrak{v i r}^{*}$ is automatically Hamiltonian with respect to the Lie-Poisson structure (8.3) and with

$$
H_{1}(m, a)=\frac{1}{2}\left(\mu(u)^{2}+\int u_{x}^{2} d x+a^{2}\right)
$$


as its Hamiltonian function, the first part of the theorem follows.

To complete the proof, observe that the corresponding Hamiltonian equation for the frozen Poisson bracket (8.4) and any function $h: \mathfrak{v i r}^{*} \rightarrow \mathbb{R}$ reads

$$
(m, a)_{t}=-a d_{\left(\frac{\delta h}{\delta m}, \frac{\delta h}{\delta a}\right)}^{*}\left(m_{0}, a_{0}\right),
$$

which, with the help of the formula (8.5) for the coadjoint action, gives

$$
\left\{\begin{array}{l}
m_{t}=-m_{0 x} \frac{\delta h}{\delta m}-2 m_{0}\left(\frac{\delta h}{\delta m}\right)_{x}-a_{0}\left(\frac{\delta h}{\delta m}\right)_{x x x} \\
a_{t}=0 .
\end{array}\right.
$$

Next, choose the Hamiltonian function $h$ to be

$$
H_{2}(m, a)=\int\left(\mu(u) u^{2}+\frac{1}{2} u u_{x}^{2}+k m u\right) d x
$$

and compute its variational derivative

$$
\frac{\delta H_{2}}{\delta m}=A^{-1}\left(\mu\left(u^{2}\right)+2 \mu(u) u-\frac{1}{2} u_{x}^{2}-u u_{x x}+k m\right),
$$

cf. equation (4.1). Substituting this expression into (8.6) and picking $\left(m_{0}, a_{0}\right)=$ $(0,-1)$ gives

$$
\left\{\begin{array}{l}
m_{t}=\partial_{x}^{3} A^{-1}\left(\mu\left(u^{2}\right)+2 \mu(u) u-\frac{1}{2} u_{x}^{2}-u u_{x x}+k m\right), \\
a_{t}=0 .
\end{array}\right.
$$

Since $\partial_{x}^{3} A^{-1}=-\partial_{x}$ and $m_{t}=-u_{t x x}$, the first of the above equations becomes

$$
-u_{t x x}=-2 \mu(u) u_{x}+2 u_{x} u_{x x}+u u_{x x x}+k u_{x x x}
$$

and a substitution $u \rightarrow u-k$ gives equation (8.1).

Remark 8.2. According to the classification given in [KM], on the Virasoro dual there are generically (i.e. at each point of the 'highest-dimensional' Virasoro coadjoint orbits) only three types of Poisson pairs consisting of the Lie-Poisson and frozen brackets. Each such pair is represented by one of the three integrable equations: $\mathrm{KdV}, \mathrm{CH}$ and HS. On the other hand, Theorem 8.1 shows that the $\mu \mathrm{HS}$ equation has the same bihamiltonian structure as $\mathrm{KdV}$ and corresponds to the same Poisson pair. How does this new integrable equation fit in the classification of $[\mathrm{KM}]$ ?

Observe that a Poisson pair determines a bihamiltonian equation only up to a choice of a Casimir (see Corollary 6.4 of $[\mathrm{KM}]$ ) and this choice is different for the $\mu \mathrm{HS}$ and the KdV equations. In fact, it is possible to choose Casimirs at each step of the Lenard-Magri scheme. In the case of $\mu \mathrm{HS}$ and $\mathrm{KdV}$ we start with the same Casimir $\int u d x$ (cf. $H_{0}$ in Section 4.1) but the subsequent choices of Casimirs are different, which results in two different integrable equations albeit having the same bihamiltonian structure.

Acknowledgments. B.K. is grateful to the IHES in Bures-sur-Yvette and the MPI in Bonn for kind hospitality. This research was partially supported by an NSERC research grant. 


\section{REFERENCES}

[ACHM] M. S. Alber, R. Camassa, D. D. Holm, and J. E. Marsden, On the link between umbilic geodesics and soliton solutions of nonlinear PDEs, Proc. Roy. Soc. London Ser. A 450 (1995), 677-692.

[Ar] V. Arnold, Sur la géometrie différentielle des groupes de Lie de dimension infinie et ses application à l'hydrodynamique des fluides parfaits, Ann. Inst. Grenoble 16 (1966), 319-361.

[AK] V. Arnold and B. Khesin, Topological Methods in Hydrodynamics, Springer, New York 1998.

[BC] A. Bressan and A. Constantin, Global solutions of the Hunter-Saxton equation, SIAM J. Math. Anal. 37 (2005), 996-1026.

[BC2] A. Bressan and A. Constantin, Global conservative solutions of the Camassa-Holm equation, Arch. Ration. Mech. Anal. 183 (2007), 215-239.

[CH] R. Camassa and D. D. Holm, An integrable shallow water equation with peaked solitons, Phys. Rev. Lett. 71 (1993), 1661-1664.

[CE] A. Constantin and J. Escher, Well-posedness, global existence and blow-up phenomena for a periodic quasi-linear hyperbolic equation, Comm. Pure Appl. Math. 51 (1998).

[CE2] A. Constantin and J. Escher, Wave breaking for nonlinear nonlocal shallow water equations, Acta Mathematica 181 (1998), 229-243.

[CKKT] A. Constantin, T. Kappeler, B. Kolev, and P. Topalov, On geodesic exponential maps of the Virasoro group, Ann. Global Anal. Geom. 31 (2007), 155- 180.

[CM] A. Constantin and H. P. McKean, A shallow water equation on the circle, Comm. Pure Appl. Math. 52 (1999), 949-982.

[EM] D. Ebin and J. E. Marsden, Groups of diffeomorphisms and the motion of an incompressible fluid, Ann. of Math. 92 (1970).

[FF] A. S. Fokas and B. Fuchssteiner, Symplectic structures, their Bäcklund transformation and hereditary symmetries, Physica D 4 (1981), 47-66.

[Gl] K. Gleason, On the periodic Hunter-Saxton equation, Senior Thesis, University of Notre Dame (2005).

[HS] J. K. Hunter and R. Saxton, Dynamics of director fields, SIAM J. Appl. Math. 51 (1991), 1498-1521.

[HZ] J. K. Hunter and Y. Zheng, On a completely integrable nonlinear hyperbolic variational equation, Physica D $\mathbf{7 9}$ (1994), 361-386.

[HZ2] J. K. Hunter and Y. Zheng, On a nonlinear hyperbolic variational equation I. Global existence of weak solutions, Arch. Rat. Mech. Anal. 129 (1995), 305-353.

[HZ3] J. K. Hunter and Y. Zheng, On a nonlinear hyperbolic variational equation II. The zero-viscosity and dispersion limits, Arch. Rat. Mech. Anal. 129 (1995), 355-383.

[J] R. Johnson, Camassa-Holm, Korteweg-de Vries and related models for water waves, $J$. Fluid Mech. 455 (2002), 63-82.

$[\mathrm{KM}]$ B. Khesin and G. Misiołek, Euler equations on homogeneous spaces and Virasoro orbits, Adv. Math. 176 (2003), 116-144.

[L] J. Lenells, Traveling wave solutions of the Camassa-Holm equation, J. Diff. Eq., 217 (2005), 393-430.

[L2] J. Lenells, The Hunter-Saxton equation describes the geodesic flow on a sphere, J. Geom. Phys. 57 (2007), 2049-2064.

[L3] J. Lenells, Weak geodesic flow and global solutions of the Hunter-Saxton equation, Disc. Cont. Dyn. Sys. 18 (2007), 643-656.

[MN] M. A. Manna and A. Neveu, A singular integrable equation from short capillary-gravity waves, preprint physics/0303085

[McK] H. P. McKean, Breakdown of a shallow water equation, Asian J. Math. 2 (1998), $867-874$.

[McK2] H. P. McKean, Compatible brackets in Hamiltonian mechanics, in "Important developments in soliton theory", pp. 344-354, Springer, Berlin, 1993. 
[M] G. Misiołek, A shallow water equation as a geodesic flow on the Bott-Virasoro group, $J$. Geom. Phys., 24 (1998), no. 3, 203-208.

[M2] G. Misiołek, Classical solutions of the periodic Camassa-Holm equation, Geom. Funct. Anal., 12 (2002), no. 5, 1080-1104.

[ON] B. O'Neill, The fundamental equations of a submersion, Michigan Math. J., 13 (1966).

[T] M. Taylor, Pseudodifferential Operators and Nonlinear PDE, Progress in Mathematics, Birkhauser Boston 1991.

[T2] M. Taylor, Finite and Infinite Dimensional Lie Groups and Evolution Equations, Lecture Notes (2003).

[Ti] F. Trğlay, The periodic Cauchy problem of the modified Hunter-Saxton equation, $J$. Evol. Equ. 5 (2005).

[Y] Z. Yin, On the structure of solutions to the periodic Hunter-Saxton equation, SIAM J. Math. Anal. 36 (2004), 272-283.

B.K.: Department of Mathematics, University of Toronto, On M5S 2E4, Canada

E-mail address: khesin@math.toronto.edu

J.L.: Department of Applied Mathematics and Theoretical Physics, University of Cambridge, Cambridge CB3 0WA, UK

E-mail address: J.Lenells@damtp.cam.ac.uk

G.M.: Department of Mathematics, University of Notre Dame, IN 46556, USA

E-mail address: gmisiole@nd.edu 